\title{
Equilibria and Free Vibration of a Two-Pulley Belt-Driven System with Belt Bending Stiffness
}

\author{
Jieyu Ding ${ }^{1}$ and Qingquan $\mathrm{Hu}^{2}$ \\ ${ }^{1}$ College of Information Engineering, Qingdao University, Qingdao 266071, China \\ ${ }^{2}$ Shandong Jiaotong University, Jinan 250023, China \\ Correspondence should be addressed to Jieyu Ding; djy@qdu.edu.cn
}

Received 20 May 2014; Revised 4 August 2014; Accepted 3 September 2014; Published 9 October 2014

Academic Editor: Francesco Pellicano

Copyright (c) 2014 J. Ding and Q. Hu. This is an open access article distributed under the Creative Commons Attribution License, which permits unrestricted use, distribution, and reproduction in any medium, provided the original work is properly cited.

\begin{abstract}
Nonlinear equilibrium curvatures and free vibration characteristics of a two-pulley belt-driven system with belt bending stiffness and a one-way clutch are investigated. With nonlinear dynamical tension, the transverse vibrations of the translating belt spans and the rotation motions of the pulleys and the accessory shaft are coupled. Therefore, nonlinear piecewise discrete-continuous governing equations are established. Considering the bending stiffness of the translating belt spans, the belt spans are modeled as axially moving beams. The pattern of equilibria is a nontrivial solution. Furthermore, the nontrivial equilibriums of the dynamical system are numerically determined by using two different approaches. The governing equations of the vibration near the equilibrium solutions are derived by introducing a coordinate transform. The natural frequencies of the dynamical systems are studied by using the Galerkin method with various truncations and the differential and integral quadrature methods. Moreover, the convergence of the Galerkin truncation is investigated. Numerical results reveal that the study needs 16 terms after truncation in order to determine the free vibration characteristics of the pulley-belt system with the belt bending stiffness. Furthermore, the first five natural frequencies are very sensitive to the bending stiffness of the translating belt.
\end{abstract}

\section{Introduction}

Pulley-belt systems play an important role in the power transmission. The vibration of pulley-belt dynamical systems greatly influences the perceived quality and the reliability of the dynamical system. Due to the complex nonlinear characteristics, pulley-belt dynamical systems have received a great deal of attention from various scholars and engineers [1-3]. Mote and $\mathrm{Wu}$ show nonlinear coupling occurs between vibration of the belt spans and wheels [4]. Kong and Parker evaluated that the influences of flexural rigidity on the equilibria [5] and the free vibration characteristics [6] of the pulley-belt dynamical system cannot be ignored. Moreover, Zhang and $\mathrm{Zu}$ found that the steady state solutions of serpentine belt drive system undergo Hopf bifurcation [7].

Pulley-belt systems in the power transmission always drive greater weight accessories, such as alternators, pumps, compressors, and fans. The driven pulley is connected to the accessories by a wrap spring. Nevertheless, the accessories with undesirable vibration transmit excessive noise and vibration to the vehicle occupants, to other vehicle structures, and may also promote the fatigue of the components of the pulley-belt systems. Moreover, the greater weight accessories may have a fatal damage to the pulley-belt system when the resonance or brake and so on behavior occur. Oneway clutches are used to erase these unfavorable influences by eliminating the opposite direction torque transmission $[8,9]$. Zhu and Parker proposed a one-way clutch model as the wrap spring with the power directional transmission function by using the harmonic balance method combined with arclength continuation [10] and the method of multiple scales [11]. The authors analyzed the nonlinear response of the dynamical system based on a piecewise two DOF discrete model and found that the discontinuous wrap spring significantly reduces resonance as an absorber. Furthermore, a simpler model by simplifying the discontinuous wrap spring as a rigidity device is proposed by Mockensturm and Balaji [12, 13]. Moreover, Gill-Jeong studied the nonlinear 
behavior of spur gear pairs with one-way clutches based on the rigidity model [14]. However, the transverse vibration of the translating belt has been ignored in all of the abovementioned literatures.

On the other hand, Wang and Mote confirmed that there is significant error in the predicted vibration behaviors if the coupling between vibration of the band spans and wheels is neglected [15]. Beikmann et al. also focused attention on a key linear mechanism that couples tensioner arm rotation and transverse vibration of the adjacent belt spans [16]. It should be mentioned that only a few attention was paid to the vibration of the pulley-belt systems coupled greater weight accessories. By using rigidity model of the one-way clutch, Zhu and Parker investigated nonlinear periodic response of three-pulley belt-driven system [17]. Furthermore, Ding and $\mathrm{Zu}$ modeled the translating belt spans as axially moving strings and found the one-way clutch significantly reduces the amplitude of the nonlinear resonance pulley-belt system [18]. However, the influences of the bending stiffness of the transport belt on the dynamics behaviors of the pulley-belt system coupled with accessories have not been understood.

In the past three decades, the translating belt in power transmission systems has been modeled as axially moving strings and beams. Jha and Parker examined eigenvalue problems in configuration space by the spatial discretization of axially moving string and beam [19]. The boundary layers [20] and the dynamics [21] of a moving belt are explored by Pellicano and his coworkers. Kong and Parker believed that the bending stiffness of the translating belt introduces nontrivial span deflections and reduces the wrap angles [22]. Dufva et al. examined the influence of the belt bending stiffness on the nonlinear vibration of a two-roller pulleybelt system and concluded that the bending stiffness cannot be in general neglected in pulley-belt applications [23]. Furthermore, Zhu and Parker investigated the influences of the dry friction on the periodic response of power transmission systems with the bending stiffness [24]. Scurtu et al. studied the resonance of the automotive serpentine belt based on a two-dimensional beam model [25].

In the present paper, a two-pulley power transmission system coupled with a one-way clutch is established by considering the bending stiffness of the translating belt. The transport belt spans are modeled as axially moving viscoelastic beams and the viscoelastic material obeys the Kelvin model. Moreover, the nontrivial span deflections and the natural frequencies are numerically studied by using the Galerkin truncation method and the differential and integral quadrature methods.

The present paper is organized into five sections. Section 2 describes the modeling of a two-pulley belt-drive dynamical system coupled with an accessory by a discontinuous wrap spring. Two different approaches for determining the nontrivial equilibrium are presented in Section 3. In Section 4, the natural frequencies of coupled vibration of the pulley-belt system are discussed, and the Galerkin truncation with various terms and the quadrature methods are compared. Section 5 ends the paper with concluding remarks.

\section{Mathematical Model}

Consider a two-pulley belt-drive dynamical system, in which the accessory shaft and the driven pulley are coupled by a wrap spring with stiffness $K_{d}$, as illustrated schematically in Figure 1, where $c$ and $P_{0}$, respectively, are the axial speed and the initial axial static tension of the translating belt and are assumed to be constant and uniform, $l$ is the length of the belt spans, $x_{i}(i=1,2)$ is the neutral axis coordinate of the $i$ th belt span, $w_{i}\left(x_{i}, t\right)(i=1,2)$ is the transverse vibration displacement of the $i$ th belt span at $x_{i}$ and time $t, \theta_{1}(t)$ and $\theta_{2}(t)$, respectively, are the angular vibration displacements of the driven pulley and the driving pulley, $M_{1}$ is the preload between the accessory shaft and the driven pulley, $J_{a}$ and $\theta_{a}(t)$ are the rotational inertia and the angular displacements of the accessory, respectively. In this work, the driving pulley and the driven pulley are assumed as the same sizes for simplicity. Furthermore, $r$ and $J$, respectively, are the radius and the rotational inertia of the pulleys. It should be noted that the accessory as a load part is rigidly connected to the shaft. Moreover, the wrap spring disconnects when angular displacement of the driven pulley is smaller than that of the accessory shaft [10], and the accessory shaft disengages from the driven pulley. Therefore, the function of power transfers in one direction of the one-way clutch is mathematically modeled by the relative angular displacement. As shown in Figure 1, there is no mechanical link between the driven pulley and the accessory shaft for disengaging state.

Considering the bending stiffness of the belt, the two spans of the translating belt are both modeled as EulerBernoulli beams. The equation of transverse motion of the belt spans is given by $[6,26]$

$$
\begin{gathered}
\rho A\left(w_{i, t t}+2 c w_{i, x_{i} t}+c^{2} w_{i, x_{i} x_{i}}\right)-P_{0} w_{i, x_{i} x_{i}} \\
+E I w_{i, x_{i} x_{i} x_{i} x_{i}}+\alpha I w_{i, x_{i} x_{i} x_{i} x_{i} t}=T_{i} w_{i, x_{i} x_{i}},
\end{gathered}
$$

where $i=1,2$, and $T_{i}$ are the dynamic tension in the above and below belt span and are defined as

$$
\begin{aligned}
& T_{1}=\frac{E A}{l} r\left(\theta_{2}-\theta_{1}\right)+\frac{E A}{2 l} \int_{0}^{l} w_{1, x_{1}}^{2} \mathrm{~d} x_{1}, \\
& T_{2}=\frac{E A}{l} r\left(\theta_{1}-\theta_{2}\right)+\frac{E A}{2 l} \int_{0}^{l} w_{2, x_{2}}^{2} \mathrm{~d} x_{2},
\end{aligned}
$$

where $\rho, E, A$, and $I$, respectively, are the density, Young's modulus, the cross-sectional area, and the area moment of inertia of the belt, $E I$ accounts for the bending stiffness, and all are assumed to be uniform. In following investigations, a rectangle-cross belt is considered. Therefore, the effect of the bending stiffness of the belt can be studied by showing the effects of Young's modulus $E$ and the height $h$. A comma preceding $x_{i}$ or $t$ denotes partial derivatives with respect to $x_{i}$ or $t$. The viscoelastic material is constituted by the Kelvin relation with the viscoelastic damping coefficient $\alpha$ [27]. The influence of the external damping of the belt is neglected in 


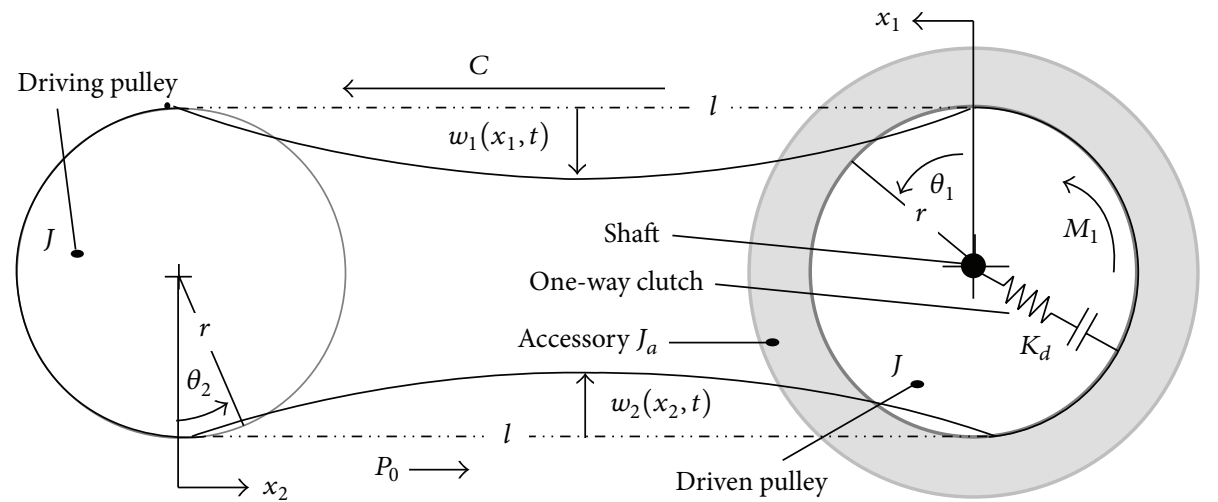

FIGURE 1: Schematic representation of a two-pulley belt-drive dynamical system coupled with a one-way clutch.

this study. The boundary conditions of the belt spans are as in the following:

$$
\begin{aligned}
& w_{i}(0, t)=0, \quad w_{i}(l, t)=0, \\
& w_{i, x_{i} x_{i}}(0, t)=w_{i, x_{i} x_{i}}(l, t)=\frac{1}{r} .
\end{aligned}
$$

The governing equation for the driving pulley, the driven pulley, and the accessory is given by

$$
\begin{gathered}
J \ddot{\theta}_{2}+c_{b} \dot{\theta}_{2}=\left(T_{2}-T_{1}\right) r, \\
J \ddot{\theta}_{1}+c_{b} \dot{\theta}_{1}=\left(T_{1}-T_{2}\right) r-f\left(\theta_{1}-\theta_{a}\right) K_{d}\left(\theta_{1}-\theta_{a}\right)+M_{1}, \\
J_{a} \ddot{\theta}_{a}+c_{a} \dot{\theta}_{a}=f\left(\theta_{1}-\theta_{a}\right) K_{d}\left(\theta_{1}-\theta_{a}\right)-M_{1},
\end{gathered}
$$

where the dot denotes differentiation with respect to $t, c_{a}$ and $c_{b}$, respectively, are the damping coefficient of the rotating of the accessory shaft and the pulleys, and piecewise function $f\left(\theta_{1}-\theta_{a}\right)$ is defined as

$$
f\left(\theta_{1}-\theta_{a}\right)= \begin{cases}1, & \theta_{1}-\theta_{a}>0 \\ 0, & \theta_{1}-\theta_{a} \leq 0 .\end{cases}
$$

It should be noted that the accessory shaft and the driven pulley remain engaged if the wrap spring without the function of power transfers in one direction. For modeling such engaged state, function $f\left(\theta_{1}-\theta_{a}\right)$ is defined as

$$
f\left(\theta_{1}-\theta_{a}\right)=1 .
$$

Incorporating the following dimensionless quantities,

$$
\begin{gathered}
x_{i} \longleftrightarrow \frac{x_{i}}{l}, \quad w_{i} \longleftrightarrow \frac{w_{i}}{l}, \\
t \longleftrightarrow t \sqrt{\frac{P_{0}}{\rho A l^{2}}}, \quad c \longleftrightarrow c \sqrt{\frac{\rho A}{P_{0}}}, \\
M_{1} \longleftrightarrow \frac{M_{1}}{P_{0} r}, \quad k_{d}=\frac{K_{d}}{P_{0} r_{a}}, \\
k_{1}=\frac{E A}{P_{0}}, \quad k_{f}=\sqrt{\frac{E I}{P_{0} l^{2}}},
\end{gathered}
$$

$$
\alpha \longleftrightarrow \frac{I \alpha}{l^{3} \sqrt{\rho A P_{0}}}, \quad c_{b} \longleftrightarrow \frac{c_{b}}{l r} \sqrt{\frac{1}{\rho A P_{0}}}
$$

$c_{a} \longleftrightarrow \frac{c_{a}}{l r_{a}} \sqrt{\frac{1}{\rho A P_{0}}}, \quad J_{a} \longleftrightarrow \frac{J_{a}}{\rho A r_{a} l^{2}}, \quad J \longleftrightarrow \frac{J}{\rho A r l^{2}}$,

the equations of motions (1) and (4) and the boundary conditions (3) can be nondimensionalized as

$$
\begin{gathered}
w_{i, t t}+2 c w_{i, x_{i} t}+\left(c^{2}-1\right) w_{i, x_{i} x_{i}} \\
+k_{f}^{2} w_{i, x_{i} x_{i} x_{i} x_{i}}+\alpha w_{i, x_{i} x_{i} x_{i} x_{i} t}=T_{i} w_{i, x_{i} x_{i}}, \\
J \ddot{\theta}_{2}=-c_{b} \dot{\theta}_{2}+T_{2}-T_{1}, \\
\ddot{\theta}_{1}=-c_{b} \dot{\theta}_{1}+T_{1}-T_{2}+M_{1} \\
-\frac{r_{a}}{r_{1}} f\left(\theta_{1}-\theta_{a}\right)\left[k_{d}\left(\theta_{1}-\theta_{a}\right)+\mu_{c}\left(\dot{\theta}_{1}-\dot{\theta}_{a}\right)\right], \\
J_{a} \ddot{\theta}_{a}=-c_{a} \dot{\theta}_{a}-\frac{r M_{1}}{r_{a}} \\
+f\left(\theta_{1}-\theta_{a}\right)\left[k_{d}\left(\theta_{1}-\theta_{a}\right)+\mu_{c}\left(\dot{\theta}_{1}-\dot{\theta}_{a}\right)\right], \\
w_{i}(0, t)=0, \quad w_{i}(1, t)=0, \\
w_{i, x_{i} x_{i}}(0, t)=w_{i, x_{i} x_{i}}(1, t)=\frac{l}{r},
\end{gathered}
$$

where

$$
\begin{aligned}
& T_{1}=\frac{k_{1} r}{l}\left(\theta_{2}-\theta_{1}\right)+\frac{k_{1}}{2} \int_{0}^{1} w_{1, x_{1}}^{2} \mathrm{~d} x_{1}, \\
& T_{2}=\frac{k_{1} r}{l}\left(\theta_{1}-\theta_{2}\right)+\frac{k_{1}}{2} \int_{0}^{1} w_{2, x_{2}}^{2} \mathrm{~d} x_{1}
\end{aligned}
$$

and $k_{1}$ and $k_{f}^{2}$, respectively, represent the effect of nonlinearity of the translating belt and the bending stiffness of the belt. 


\section{The Nontrivial Equilibrium}

In this section, the nontrivial equilibrium is determined via two different ways. At first, the equilibria of the dynamical system are achieved as asymptotic behaviors of a viscoelastic model. Then an iterative scheme is developed for confirming the asymptotic solution.

3.1. The Nontrivial Solutions of Viscoelastic Model. The differential and integral quadrature methods have been applied to study the nonlinear dynamics of continua $[26,28,29]$. In the following, the quadrature methods are applied to numerically calculate the nontrivial equilibrium solution.

In the domain of $x_{i}, N$ is introduced as Chebyshev-GaussLobatto sampling points with $\delta$ points immediate adjacent at both ends for the two belt spans as

$$
\begin{gathered}
x_{i j}=\frac{1}{2}\left[1-\cos \frac{(j-2) \pi}{N-3}\right], \quad j=3,4, \ldots, N-2, \\
x_{i 1}=0, \quad x_{i 2}=\delta, \quad x_{i(N-1)}=1-\delta, \quad x_{i N}=1,
\end{gathered}
$$

where $i=1$ and 2. By the quadrature rule, an $n$ th-order derivative at $x_{i j}$ and the integral terms in (10), respectively, are written as

$$
\begin{gathered}
\left.\frac{\partial^{n} g\left(x_{i}\right)}{\partial x_{i}^{n}}\right|_{x_{i}=x_{i j}}=\sum_{k=1}^{N} A_{j k}^{(n)} g_{i k}, \\
\int_{0}^{1} w_{i, x_{i}}^{2} \mathrm{~d} x_{i}=\sum_{g=1}^{N} I_{g}\left[\sum_{j=1}^{N} A_{g j}^{(1)} w_{i j}\right]^{2}, \quad i=1,2,
\end{gathered}
$$

where $g\left(x_{i}\right)$ is an arbitrary function, $g_{i k}$ represents $g\left(x_{i}\right)$ on the sampling point $x_{i k}$, and $A_{j k}^{(n)}$ and $I_{g}$ represent the quadrature weighting coefficients and the integral weighting coefficients, respectively [26].

Substitution of (12) into (8) and (9) yields a series of ordinary differential equations

$$
w_{i}\left(x_{i 1}, t\right)=0 \text {, }
$$

$$
\begin{aligned}
& w_{i}\left(x_{i 2}, t\right) \\
& =\frac{\left[l / r-A_{2(N-1)}^{(2)} w_{i(N-1)}-\sum_{k=3}^{N-2} A_{2 k}^{(2)} w_{i}\left(x_{i k}, t\right)\right]}{A_{22}^{(2)}}, \\
& \ddot{w}_{i}\left(x_{i j}, t\right)-\sum_{k=1}^{N}\left[\left(\gamma^{2}-1\right) A_{j k}^{(2)}+k_{f}^{2} \widetilde{A}_{j k}^{(4)}\right] w_{i}\left(x_{i k}, t\right) \\
& +\sum_{k=1}^{N}\left[2 \gamma A_{j k}^{(1)}+\alpha A_{j k}^{(4)}\right] \dot{w}_{i}\left(x_{i k}, t\right)
\end{aligned}
$$

$$
\begin{array}{r}
=\frac{k_{1}^{2}}{2}\left[\sum_{k=1}^{N} A_{j k}^{(2)} w_{i}\left(x_{i k}, t\right)\right] \\
\times\left\{\lambda_{i}+\sum_{g=1}^{N} I_{g}\left[\sum_{k=1}^{N} A_{g k}^{(1)} w_{i}\left(x_{i k}, t\right)\right]^{2}\right\}, \\
j=3,4, \ldots, N-2,
\end{array}
$$

$w_{i}\left(x_{i(N-1)}, t\right)$

$$
\begin{aligned}
=\left(\frac{l}{r}-\right. & \sum_{k=3}^{N-2} A_{(N-1) k}^{(2)} w_{i}\left(x_{i k}, t\right) \\
& \left.-\frac{A_{(N-1) 2}^{(2)}\left[l / r-\sum_{k=3}^{N-2} A_{2 k}^{(2)} w_{i}\left(x_{i k}, t\right)\right]}{A_{22}^{(2)}}\right)
\end{aligned}
$$$$
\times\left(A_{(N-1)(N-1)}^{(2)}-\frac{A_{(N-1) 2}^{(2)} A_{2(N-1)}^{(2)}}{A_{22}^{(2)}}\right)^{-1},
$$$$
w_{i}\left(x_{i N}, t\right)=0,
$$

$$
\begin{aligned}
J \ddot{\theta}_{2}= & -c_{b} \dot{\theta}_{2}+\frac{k_{1}}{2} \\
& \times\left\{\frac{4 r}{l}\left(\theta_{1}-\theta_{2}\right)+\sum_{g=1}^{N} I_{g}\left[\sum_{k=1}^{N} A_{g k}^{(1)} w_{2}\left(x_{2 k}, t\right)\right]^{2}\right. \\
J \ddot{\theta}_{1}= & \left.-c_{b} \dot{\theta}_{1}+\frac{k_{1}}{2} \sum_{g=1}^{N}\left[\sum_{k=1}^{N} A_{g k}^{(1)} w_{1}\left(x_{1 k}, t\right)\right]^{2}\right\}, \\
& \times\left\{\frac{4 r}{l}\left(\theta_{2}-\theta_{1}\right)+\sum_{g=1}^{N} I_{g}\left[\sum_{k=1}^{N} A_{g k}^{(1)} w_{1}\left(x_{1 k}, t\right)\right]^{2}\right. \\
& \quad \times\left[K_{d}\left(\theta_{1}-\theta_{a}\right)+\mu_{d}\left(\dot{\theta}_{1}-\dot{\theta}_{a}\right)\right], \\
& \left.+\sum_{a} I_{a} I_{g}\left[\sum_{k=1}^{N} A_{g k}^{(1)} w_{2}\left(x_{2 k}, t\right)\right]^{2}\right\} \\
& \times\left[K_{d}\left(\theta_{1}-\theta_{a}\right)+\mu_{d}\left(\dot{\theta}_{1}-\dot{\theta}_{a}\right)\right] \frac{r M_{1}}{r}, f\left(\theta_{1}-\theta_{a}\right)
\end{aligned}
$$

where $i=1,2$, and

$$
\lambda_{1}=\frac{2 r}{l}\left(\theta_{2}-\theta_{1}\right), \quad \lambda_{2}=\frac{2 r}{l}\left(\theta_{1}-\theta_{2}\right) .
$$


The physical and geometrical properties of the two-pulley belt-driven system are listed in Table $1[10,18,30,31]$.

Based on mesh grid (11) with $\delta=0.00001$, the numerical solution is solved from (13) by discretizing the temporal variables with the fixed temporal step $10^{-4}$. Besides, the initial conditions for the first calculation are the same for all the following calculations as given below

$$
\begin{gathered}
w_{i}\left(x_{i j}, 0\right)=D_{m} \sin \left(\pi x_{i j}\right), \\
w_{i, t}\left(x_{i j}, 0\right)=0,
\end{gathered}
$$

where $i=1$ and $2, j=1,2, \ldots, N$, and $D_{m}$ represents the amplitude of the belt span's vibration. It should be noted that $D_{m}=0.001$ is used in all numerical examples. Furthermore, $N=17$ in the following computations if there is no statement.

The transverse displacements of the center of the translating belt spans via the quadrature methods with the boundary condition (9) and the initial condition (15) are plotted in Figure 2 with the axial transporting speed of the belt $c=21.43 \mathrm{~m} / \mathrm{s}$. In Figure 2(a), the numerical simulations demonstrate that the vibration responses of the belt spans depend on the initial conditions at the beginning phase, then the vibration response gradually decays, and a nontrivial equilibrium of the belt forms finally. Therefore, Figure 2 illustrates that the nontrivial equilibrium solutions can be obtained from the free vibration of the viscoelastic model by using the quadrature methods.

3.2. The Iterative Solution. The nontrivial equilibrium solutions $\widehat{w}_{1}\left(x_{1}\right)$ and $\widehat{w}_{2}\left(x_{2}\right)$ of (8) in engaged state satisfy

$$
\begin{gathered}
\left(c^{2}-1-\widehat{T}\right) \widehat{w}_{i}^{\prime \prime}+k_{f}^{2} \widehat{w}_{i}^{\prime \prime \prime \prime}=0, \quad i=1,2, \\
-M_{1}+\frac{r_{a}}{r}\left[k_{d}\left(\widehat{\theta}_{1}-\widehat{\theta}_{a}\right)\right]=0,
\end{gathered}
$$

where

$$
\widehat{T}=\frac{k_{1}}{4}\left(\int_{0}^{1} \widehat{w}_{1}^{\prime 2} \mathrm{~d} x_{1}+\int_{0}^{1} \widehat{w}_{2}^{\prime 2} \mathrm{~d} x_{2}\right) .
$$

The boundary conditions of the two transporting belt spans for (16) are as follows:

$$
\begin{gathered}
\widehat{w}_{i}(0)=0, \quad \widehat{w}_{i}(1)=0, \\
\widehat{w}_{i}^{\prime \prime}(0)=\widehat{w}_{i}^{\prime \prime}(1)=\frac{l}{r} .
\end{gathered}
$$

Substitution of (12) into (16) and (18) yields a series of algebraic equations

$$
\begin{gathered}
\left(c^{2}-1-\widehat{T}\right) \sum_{k=1}^{N} A_{j k}^{(2)} \widehat{w}_{i k}+k_{f}^{2} \sum_{k=1}^{N} A_{j k}^{(4)} \widehat{w}_{i k}=0, \\
j=3,4, \ldots, N-2, \\
\widehat{w}_{i 1}=\widehat{w}_{i N}=0, \\
\sum_{k=1}^{N} A_{2 k}^{(2)} \widehat{w}_{i k}=\sum_{k=1}^{N} A_{(N-1) k}^{(2)} \widehat{w}_{i k}=\frac{l}{r},
\end{gathered}
$$

TABLE 1: Properties of the two-pulley belt-driven system with a oneway clutch.

\begin{tabular}{lcc}
\hline Item & Notation & Value \\
\hline Radius & Pulleys & \\
Preload on driven pulley & $r$ & $0.0452 \mathrm{~m}$ \\
Rotation inertia & $M_{1}$ & $5 \mathrm{~N} \mathrm{~m}$ \\
Damping coefficient & $J$ & $0.001607 \mathrm{~kg} \mathrm{~m}{ }^{2}$ \\
\hline & $c_{b}$ & $0.02 \mathrm{~N} \mathrm{~m} \mathrm{~s}$ \\
Radius & Accessory & \\
Rotation inertia & $r_{a}$ & $0.0889 \mathrm{~m}$ \\
Damping coefficient & $J_{a}$ & $0.002603 \mathrm{~kg} \mathrm{~m}$ \\
\hline & $c_{a}$ & $0.02 \mathrm{~N} \mathrm{~m} \mathrm{~s}$ \\
Length of span & Belt & \\
Young's modulus & $l$ & $0.5518 \mathrm{~m}$ \\
Width & $E$ & $2 \times 10^{9} \mathrm{~Pa}$ \\
Height & $b$ & $0.02 \mathrm{~m}$ \\
Density & $h$ & $0.005 \mathrm{~m}$ \\
Static tension & $\rho$ & $1150 \mathrm{~kg} / \mathrm{m}^{3}$ \\
Viscous damping & $P_{0}$ & $350 \mathrm{~N}$ \\
& $\alpha$ & $2 \times 10^{6} \mathrm{~N} \mathrm{~s} / \mathrm{m}^{2}$ \\
Torsional stiffness & One-way clutch & $4000 \mathrm{~N} \mathrm{~m}$ \\
\hline
\end{tabular}

where $i=1$ and 2. Similarly, substitution of (12) into (17) yields

$$
\begin{aligned}
\widehat{T}=\frac{k_{1}}{4} & \\
& \times\left\{\sum_{g=1}^{N}\left\{I_{g}\left[\sum_{k=1}^{N} A_{g k}^{(1)} \widehat{w}_{1 k}\right]^{2}\right\}\right. \\
& \left.+\sum_{g=1}^{N}\left\{I_{g}\left[\sum_{k=1}^{N} A_{g k}^{(1)} \widehat{w}_{2 k}\right]^{2}\right\}\right\} .
\end{aligned}
$$

For solving the algebraic equations (19), an iterative scheme is developed as follows:

$$
\begin{gathered}
\widehat{w}_{i j}=\left(-\left[\left(c^{2}-1-\widehat{T}\right) \sum_{k=1, k \neq j}^{N} A_{j k}^{(2)}\right.\right. \\
\left.\left.+k_{f}^{2} \sum_{k=1, k \neq j}^{N} A_{j k}^{(4)}\right] \widehat{w}_{i k}\right) \\
\times\left(\left(c^{2}-1-\widehat{T}\right) A_{j j}^{(2)}+k_{f}^{2} A_{j j}^{(4)}\right)^{-1} \\
j=3,4, \ldots, N-2, \\
\widehat{w}_{i 1}=\widehat{w}_{i N}=0, \\
\widehat{w}_{i j}=\frac{l / r-\sum_{k=1, k \neq j}^{N} A_{j k}^{(2)} \widehat{w}_{i k}}{A_{j j}^{(2)}}, \quad j=2, N-1 .
\end{gathered}
$$




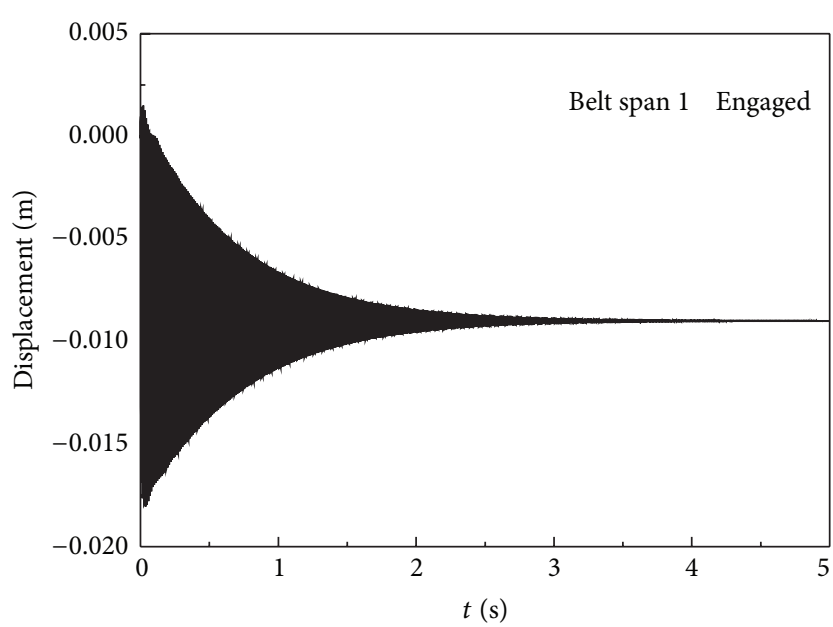

(a) Belt span 1

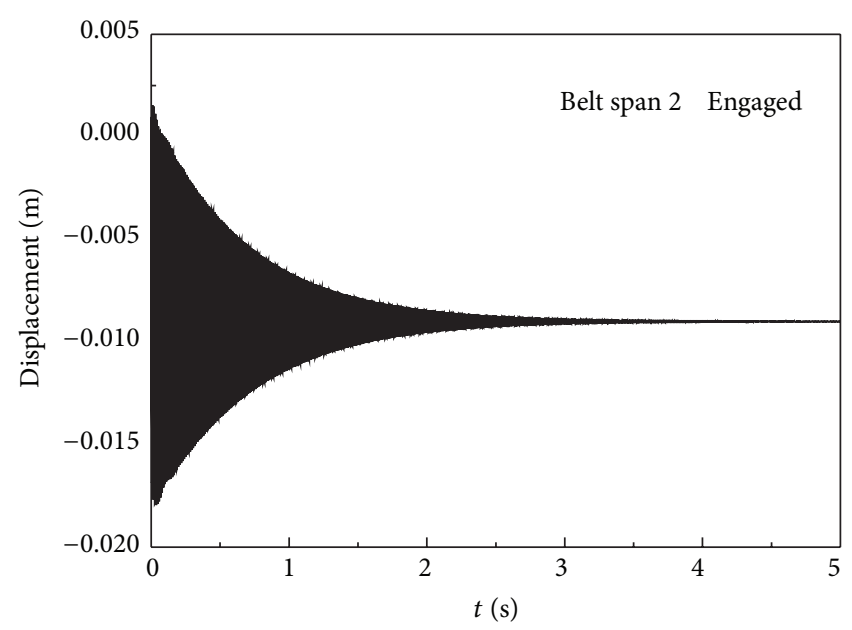

(b) Belt span 2

FIGURE 2: The time history calculated from (13) via the quadrature methods.

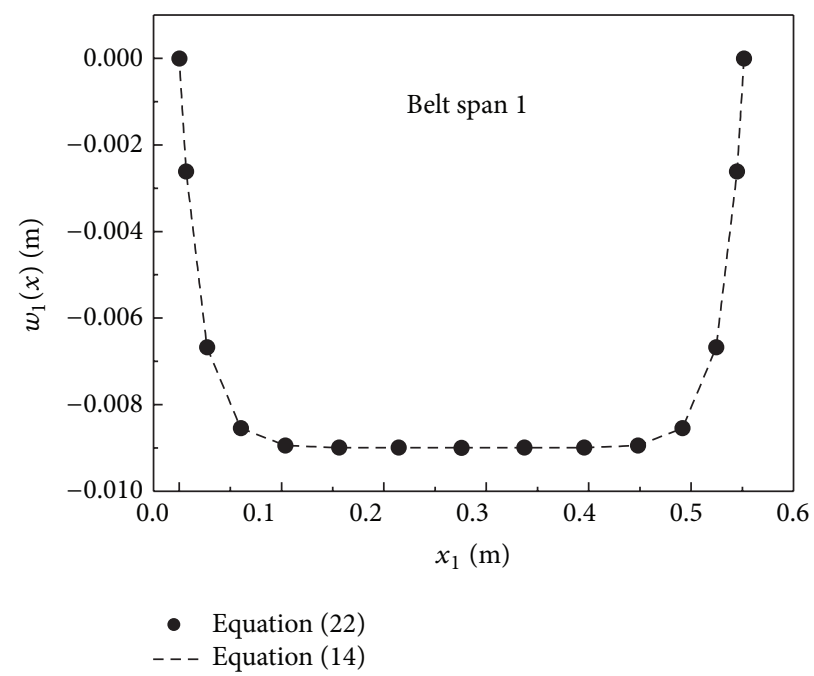

(a) Belt span 1

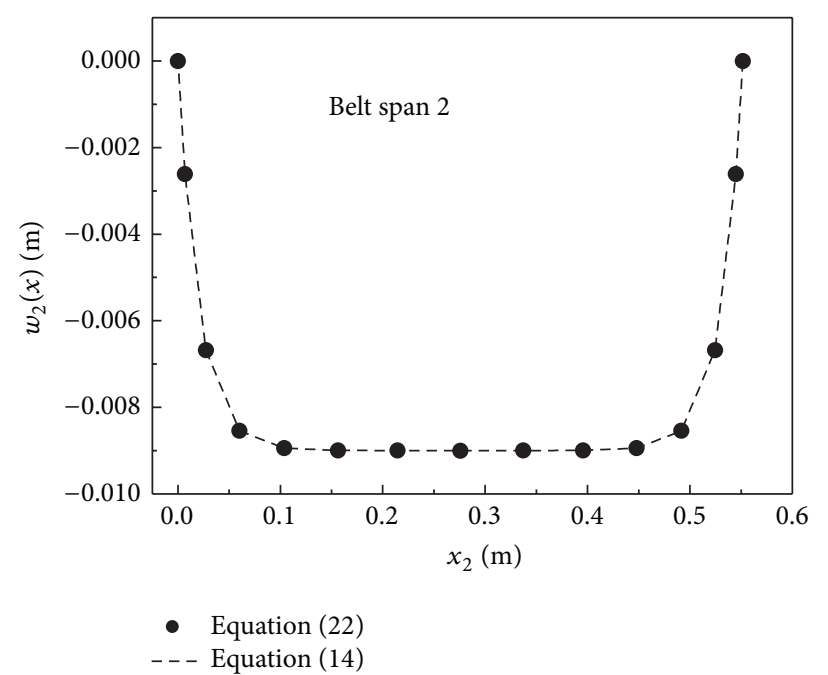

(b) Belt span 2

FIGURE 3: The comparison of the nontrivial equilibrium calculated from (13) and (21).

Figure 3 shows the comparison of the nontrivial equilibrium between iterative scheme (21) and the free vibration of the viscoelastic model (13). In Figure 3, the number of iteration $D=100000$. Figures 3(a) and 3(b), respectively, depict the comparison of the nontrivial equilibrium of the translating belt span 1 and span 2. As shown in Figure 3, the nontrivial equilibria calculated from two different ways are completely coincident. Therefore, the nontrivial equilibrium solutions of the two-pulley belt-driven system are efficiently determined by using the iterative procedure in conjunction with the differential and integral quadrature methods. In the following investigation, the nontrivial equilibria are all calculated by iterative scheme (21).

The effects of the physical parameters, the speed, and the initial static tension are shown in Figure 4 with the axial speed of the belt $c=21.43 \mathrm{~m} / \mathrm{s}$. It should be noted that here we only show the nontrivial equilibrium solutions of the belt span 1 , as the results of the belt span 2 are completely the same. With the increase of the length of the belt span, the axial speed of the belt, the height of the belt, Young's modulus of the belt, and the nontrivial equilibrium solutions increase. Meanwhile, with the increase of the radius of the driving pulley and the driven pulley and the initial static tension in the translating belt, the nontrivial equilibrium decreases. Since EI accounts for the belt bending stiffness, Figures 4(d) and $4(\mathrm{e})$ illustrate that the nontrivial equilibrium solutions are very sensitive to the belt bending stiffness.

\section{Natural Frequencies}

Substitution $u_{i}\left(x_{i}, t\right)+\widehat{w}_{i}\left(x_{1}\right) \rightarrow w_{i}\left(x_{i}, t\right), \vartheta_{i}(t)+\widehat{\theta}_{i} \rightarrow \theta_{i}(t)$, and $\vartheta_{a}(t)+\hat{\theta}_{a} \rightarrow \theta_{a}(t)$ in (8) and (9) in engaged state, where 

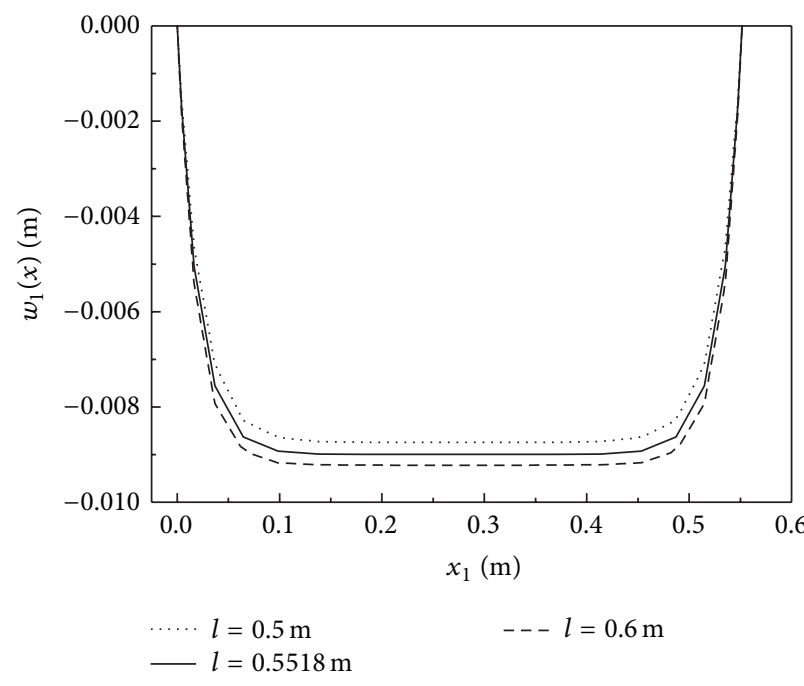

(a) The effect of the length of the belt span

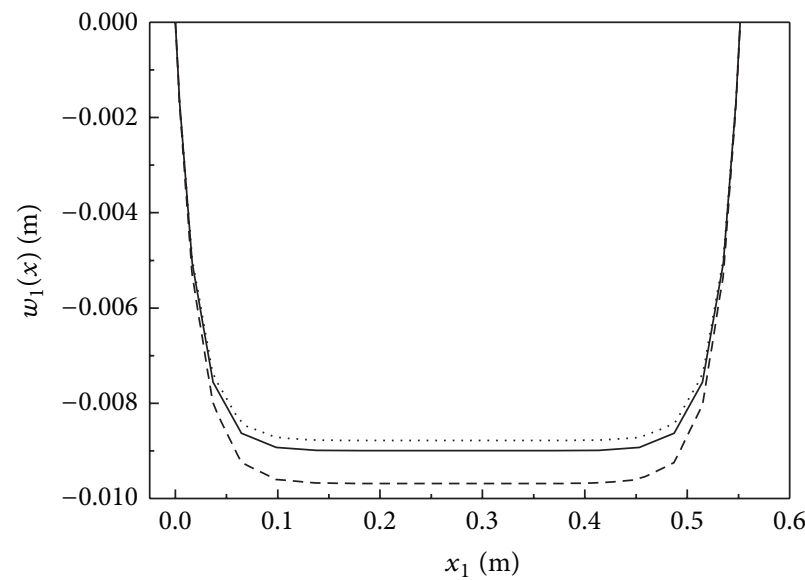

$c=5.36 \mathrm{~m} / \mathrm{s}$

$-c=26.79 \mathrm{~m} / \mathrm{s}$

(c) The effect of the speed of the belt

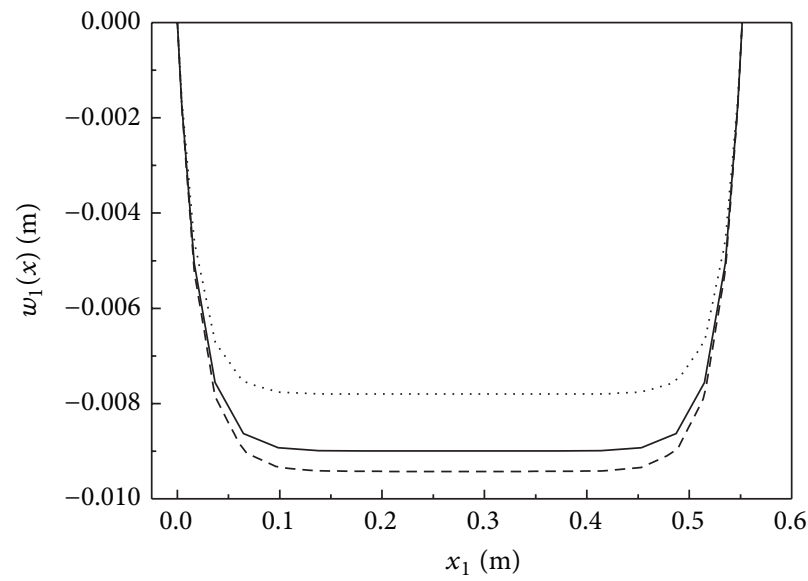

$\begin{aligned} E & =1 * 10^{9} \mathrm{~N} / \mathrm{m}^{2} \\ E & =2 * 10^{9} \mathrm{~N} / \mathrm{m}^{2}\end{aligned}$

(e) The effect of the Young's modulus of the belt

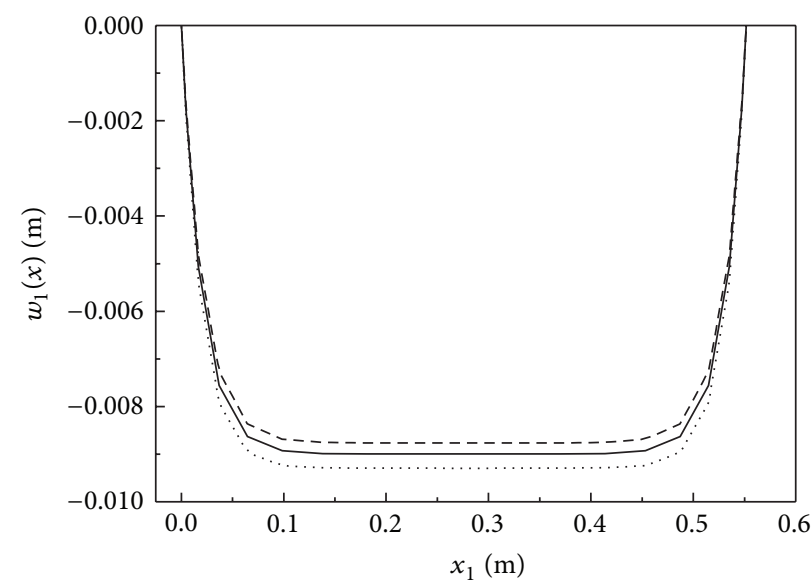

$$
\begin{aligned}
r & =0.04 \mathrm{~m} \\
-r & =0.0452 \mathrm{~m}
\end{aligned}
$$$$
---r=0.05 \mathrm{~m}
$$

(b) The effect of the radius of the pulleys

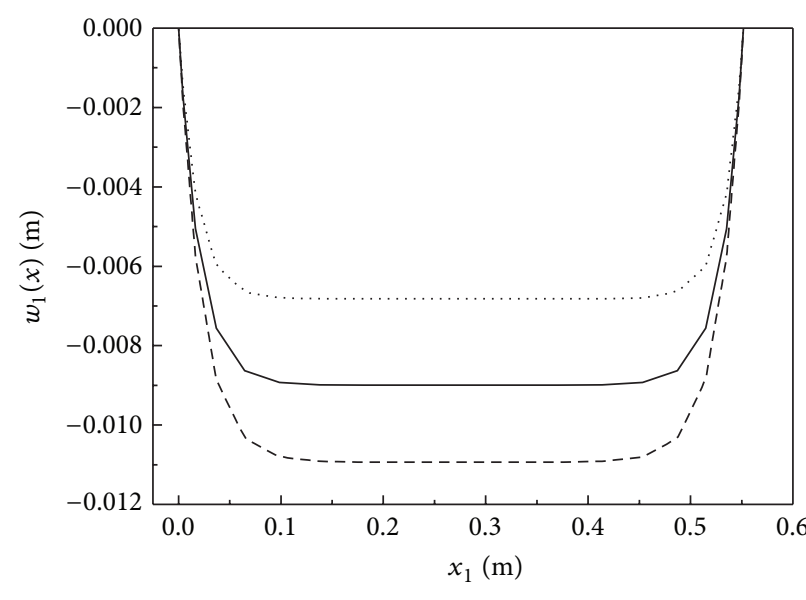

$$
\begin{aligned}
\cdots \cdots h & =0.004 \mathrm{~m} \\
-h & =0.005 \mathrm{~m}
\end{aligned}
$$

(d) The effect of the belt's height

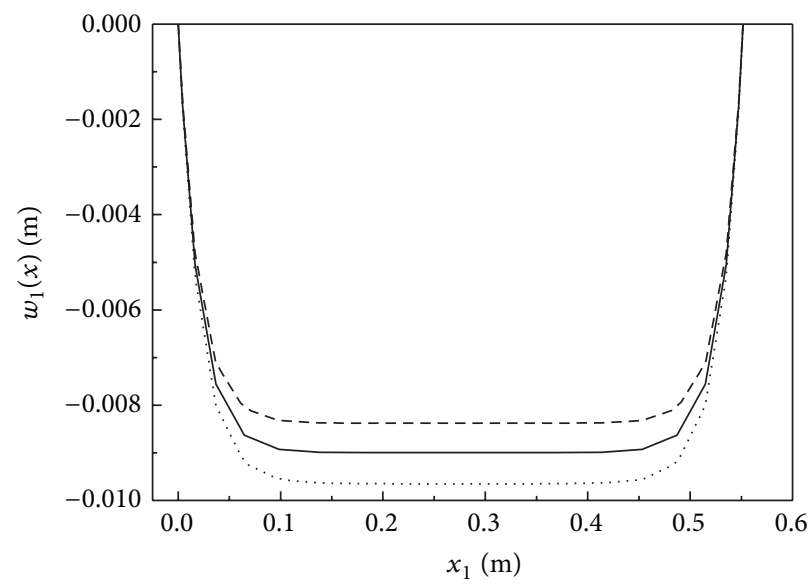

$\begin{aligned} \cdots P_{0} & =200 \mathrm{~N} \\ -P_{0} & =350 \mathrm{~N}\end{aligned}$

(f) The effect of the initial static tension

FIGURE 4: The parametric studies for the using of nontrivial equilibrium solutions via iterative procedure. 
$i=1,2$, yields the governing equation of motion measured from the nontrivial equilibrium $[32,33]$

$$
\begin{aligned}
u_{i, t t}+ & 2 c u_{i, x_{i} t}+\left(c^{2}-1-\widehat{T}-k_{1} \int_{0}^{1} \widehat{w}_{i}^{\prime} u_{i} x_{i} \mathrm{~d} x_{i}-P_{i}\right) u_{i, x_{i} x_{i}} \\
& +k_{f}^{2} u_{i, x_{i} x_{i} x_{i} x_{i}}+\alpha u_{i, x_{i} x_{i} x_{i} x_{i} t} \\
= & \left(k_{1} \int_{0}^{1} \widehat{w}_{i}^{\prime} u_{i, x_{i}} \mathrm{~d} x_{i}+P_{i}\right) \widehat{w}_{i}^{\prime \prime}, \\
J \ddot{\vartheta}_{1}= & -c_{b} \dot{\vartheta}_{1}+P_{1}-P_{2}+k_{1} \int_{0}^{1} \widehat{w}_{1}^{\prime} u_{1, x_{1}} \mathrm{~d} x_{1} \\
& -k_{1} \int_{0}^{1} \widehat{w}_{2}^{\prime} u_{2, x_{2}} \mathrm{~d} x_{2}-f\left(\vartheta_{1}-\vartheta_{a}+\frac{r M_{1}}{r_{a} k_{d}}\right) \\
& \times\left[k_{d}\left(\vartheta_{1}-\vartheta_{a}+\frac{r M_{1}}{r_{a} k_{d}}\right)+\mu_{c}\left(\dot{\vartheta}_{1}-\dot{\vartheta}_{a}\right)\right] \frac{r_{a}}{r}+M_{1}, \\
& J \ddot{\vartheta}_{2}=-c_{b} \dot{\vartheta}_{2}+P_{2}-P_{1}, \\
J_{a} \ddot{\vartheta}_{a}= & -c_{a} \dot{\vartheta}_{a}+f\left(\vartheta_{i, x_{i} x_{i}}(0, t)=\vartheta_{i, x_{i} x_{i}}(1, t)=0, \frac{r M_{1}}{r_{a} k_{d}}\right) \\
& \times\left[k_{d}\left(\vartheta_{1}-\vartheta_{a}+\frac{r M_{1}}{r_{a} k_{d}}\right)+\mu_{c}\left(\dot{\vartheta}_{1}-\dot{\vartheta}_{a}\right)\right]-\frac{r M_{1}}{r_{a}} \\
& \\
& \\
&
\end{aligned}
$$

where

$$
\begin{aligned}
& P_{1}=\frac{k_{1} r}{l}\left(\vartheta_{2}-\vartheta_{1}\right)+\frac{k_{1}}{2} \int_{0}^{1} u_{1, x_{1}}^{2} \mathrm{~d} x_{1}, \\
& P_{2}=\frac{k_{1} r}{l}\left(\vartheta_{1}-\vartheta_{2}\right)+\frac{k_{1}}{2} \int_{0}^{1} u_{2, x_{2}}^{2} \mathrm{~d} x_{2} .
\end{aligned}
$$

The Galerkin truncation will be used to numerically solve the linear equations, correspondingly those nonlinear equations for the natural frequencies under the boundary conditions (23). Omitting damping terms, the nonlinear terms of (22) yield the following linear form with space-dependent coefficients

$$
\begin{aligned}
u_{i, t t}+ & 2 c u_{i, x_{i} t}-\left(k_{1} \int_{0}^{1} \widehat{w}_{i}^{\prime} u_{i, x_{i}} \mathrm{~d} x_{i}+\widetilde{\lambda}_{i}\right) \widehat{w}_{i}^{\prime \prime} \\
& +\left(c^{2}-1-\widehat{T}\right) u_{i, x_{i} x_{i}}+k_{f}^{2} u_{i, x_{i} x_{i} x_{i} x_{i}}=0, \\
J \ddot{\vartheta}_{1}= & \frac{2 k_{1} r}{l}\left(\vartheta_{2}-\vartheta_{1}\right)-k_{d}\left(\vartheta_{1}-\vartheta_{a}\right) \frac{r_{a}}{r} \\
& +k_{1} \int_{0}^{1} \widehat{w}_{1}^{\prime} u_{1, x_{1}} \mathrm{~d} x_{1}-k_{1} \int_{0}^{1} \widehat{w}_{2}^{\prime} u_{2, x_{2}} \mathrm{~d} x_{2},
\end{aligned}
$$

$$
\begin{gathered}
J \ddot{\vartheta}_{2}=\frac{2 k_{1} r}{l}\left(\vartheta_{1}-\vartheta_{2}\right) \\
+k_{1}\left(\int_{0}^{1} \widehat{w}_{2}^{\prime} u_{2, x_{2}} \mathrm{~d} x_{2}-\int_{0}^{1} \widehat{w}_{1}^{\prime} u_{1, x_{1}} \mathrm{~d} x_{1}\right), \\
J_{a} \ddot{\vartheta}_{a}=k_{d}\left(\vartheta_{1}-\vartheta_{a}\right),
\end{gathered}
$$

where

$$
\begin{aligned}
& \tilde{\lambda}_{1}=\frac{k_{1} r}{l}\left(\vartheta_{2}-\vartheta_{1}\right), \\
& \tilde{\lambda}_{2}=\frac{k_{1} r}{l}\left(\vartheta_{1}-\vartheta_{2}\right) .
\end{aligned}
$$

4.1. Galerkin Discretization. The Galerkin truncation will be proposed to discretize the equation of motion of the twopulley belt-driven system into ordinary differential equations. In the present investigation, both the trial and weight functions are chosen as eigenfunctions of a linear nontranslating beam under the boundary condition (23); namely, suppose that the solution of $(25)$ takes the form $[6,34]$

$$
\begin{aligned}
u_{i}\left(x_{i}, t\right)=\sum_{j=1}^{n} q_{i j} e^{i \omega t} \sin \left(j \pi x_{i}\right), \quad j=1,2, \ldots, n ; i=1,2, \\
\vartheta_{1}=Q_{1} e^{i \omega t} \\
\vartheta_{2}=Q_{2} e^{i \omega t} \\
\vartheta_{a}=Q_{a} e^{i \omega t}
\end{aligned}
$$

where $q_{i j}(t)(i=1,2 ; j=1,2, \ldots, n)$ is the $j$ th modal coordinates for the $i$ th belt span. After substituting (27) into (25), the Galerkin procedure leads to the following set of second-order ordinary differential equations

$$
\begin{aligned}
& -\omega^{2} \sum_{j=1}^{n} q_{i j} \sin \left(j \pi x_{i}\right)+i 2 c \omega \sum_{j=1}^{n} j \pi q_{i j} \cos \left(j \pi x_{i}\right) \\
& +\left(c^{2}-1-\widehat{T}\right) \sum_{j=1}^{n}\left[-(j \pi)^{2} q_{i j} \sin \left(j \pi x_{i}\right)\right] \\
& -\left[k_{1} \int_{0}^{1} \widehat{w}_{i, x_{i}} \sum_{j=1}^{n} j \pi q_{i j} \cos \left(j \pi x_{i}\right) \mathrm{d} x_{i}+\widehat{\lambda}_{i}\right] \widehat{w}_{i}^{\prime \prime} \\
& +k_{f}^{2} \sum_{j=1}^{n}(j \pi)^{4} q_{i j} \sin \left(j \pi x_{i}\right)=0, \\
& -\omega^{2} J Q_{1}-\frac{2 r k_{1}}{l}\left(Q_{2}-Q_{1}\right)+k_{d}\left(Q_{1}-\theta_{a}\right) \frac{r_{a}}{r} \\
& \quad-k_{1} \int_{0}^{1} \widehat{w}_{1}^{\prime} \sum_{j=1}^{n} j \pi q_{1 j} \cos \left(j \pi x_{1}\right) \mathrm{d} x_{1} \\
& +k_{1} \int_{0}^{1} \widehat{w}_{2}^{\prime} \sum_{j=1}^{n} j \pi q_{2 j} \cos \left(j \pi x_{2}\right) \mathrm{d} x_{2}=0,
\end{aligned}
$$




$$
\begin{aligned}
& -\omega^{2} J Q_{2}-\frac{2 k_{1} r}{l}\left(Q_{1}-Q_{2}\right)-k_{1} \\
& \quad \times \int_{0}^{1} \widehat{w}_{2}^{\prime} \sum_{j=1}^{n} j \pi q_{2 j} \cos \left(j \pi x_{2}\right) \mathrm{d} x_{2} \\
& +k_{1} \int_{0}^{1} \widehat{w}_{1}^{\prime} \sum_{j=1}^{n} j \pi q_{1 j} \cos \left(j \pi x_{1}\right) \mathrm{d} x_{1}=0, \\
& -\omega^{2} J_{a} Q_{a}-k_{d}\left(Q_{1}-\theta_{a}\right)=0,
\end{aligned}
$$

where

$$
\begin{aligned}
& \hat{\lambda}_{1}=\frac{k_{1} r}{l}\left(Q_{2}-Q_{1}\right), \\
& \hat{\lambda}_{2}=\frac{k_{1} r}{l}\left(Q_{1}-Q_{2}\right) .
\end{aligned}
$$

Multiplying the first equation of (28) by weighted function $\sin \left(m \pi x_{i}\right)$ and integrating the product from 0 to 1 yield

$$
\begin{gathered}
-\frac{1}{2} \omega^{2} q_{i m}+i \omega \sum_{j=1}^{n} 2 c G_{m j}^{i} q_{i j} \\
+\frac{1}{2}(m \pi)^{2}\left[(m \pi)^{2} k_{f}^{2}-c^{2}+1+\widehat{T}\right] q_{i m} \\
-k_{1} P_{i m} \sum_{j=1}^{n} R_{i j} q_{i j}+P_{i m} \hat{\lambda}_{i}=0, \\
-\omega^{2} J Q_{1}-k_{1} \sum_{j=1}^{n} R_{1 j} q_{1 j}+k_{1} \sum_{j=1}^{n} R_{2 j} q_{2 j} \\
+\left(\frac{2 r k_{1}}{l}+\frac{r_{a} k_{d}}{r}\right) Q_{1}-\frac{2 r k_{1}}{l} Q_{2}-\frac{r_{a} k_{d}}{r} Q_{a}=0, \\
-\omega^{2} J Q_{2}+k_{1} \sum_{j=1}^{n} R_{1 j} q_{1 j}-k_{1} \sum_{j=1}^{n} R_{2 j} q_{2 j} \\
-\frac{2 k_{1} r}{l} Q_{1}+\frac{2 k_{1} r}{l} Q_{2}=0, \\
-\omega^{2} J_{a} Q_{a}-k_{d} Q_{1}+k_{d} Q_{a}=0,
\end{gathered}
$$

where

$$
\begin{gathered}
R_{1 j}=j \pi \int_{0}^{1} \widehat{w}_{1}^{\prime} \cos \left(j \pi x_{1}\right) \mathrm{d} x_{1}, \\
R_{2 j}=\int_{0}^{1} \widehat{w}_{2}^{\prime}\left[j \pi \cos \left(j \pi x_{2}\right)\right] \mathrm{d} x_{2}, \\
P_{1 m}=\int_{0}^{1} \widehat{w}_{1}^{\prime \prime} \sin \left(i \pi x_{1}\right) \mathrm{d} x_{1}, \\
P_{2 m}=\int_{0}^{1} \widehat{w}_{2}^{\prime \prime} \sin \left(i \pi x_{2}\right) \mathrm{d} x_{2}, \\
G_{m j}^{i}=\int_{0}^{1} j \pi \cos \left(j \pi x_{i}\right) \sin \left(m \pi x_{i}\right) \mathrm{d} x_{i},
\end{gathered}
$$

where $i=1,2 ; m=1,2, \ldots, n ; j=1,2, \ldots, n$. Equation $(30)$ can be written in matrix-vector form as

$$
\left[-\omega^{2} \mathbf{M}+i \omega \mathbf{G}+\mathbf{K}\right] \mathbf{Q}=0,
$$

where

$$
\mathbf{Q}=\left(\begin{array}{lllllllllll}
q_{11} & q_{12} & \cdots & q_{1 n} & q_{21} & q_{22} & \cdots & q_{2 n} & Q_{1} & Q_{2} & Q_{a}
\end{array}\right)^{T}
$$

and all coefficients $M_{h k}, G_{h k}$, and $K_{h k}, h, k=1,2, \ldots, 2 n+$ 3 are determined by the Galerkin procedure [24, 26, 35]. Nontriviality of solutions to (32) requires its determinant of coefficients to be zero; therefore, the natural frequencies $\omega$ of the dynamical system can be obtained from

$$
\left|-\omega^{2} \mathbf{M}+i \omega \mathbf{G}+\mathbf{K}\right|=0 .
$$

4.1.1. Convergence Study. Figure 5 shows the comparisons for the first five natural frequencies of the two-pulley beltdrive dynamical system between different truncation terms. In Figure 5, the natural frequencies are calculated for the different speed of the transport belt. As it is seen from Figures 5(a) and 5(b), the third, fourth, and fifth natural frequencies in engaged state via 6-term Galerkin truncation are bigger than that of 16-term Galerkin method. Meanwhile, the difference of the first two natural frequencies of the dynamical system between 6-term and 16-term Galerkin truncation is quite small. Particularly, the first natural frequencies via 6term and 16-term Galerkin truncation are almost coincident. Furthermore, the difference between the fourth and fifth natural frequencies via 6-term Galerkin truncation and 16term Galerkin method is increased with increasing belt transport speed. However, the quantitative difference of the fourth and fifth natural frequencies between 8-term, 10-term, 12-term, and 16-term Galerkin method decreases as shown in Figures 6(d), 6(f), and 6(h). Furthermore, the comparison in Figure 6 demonstrates that 16-term Galerkin truncation method obtains the convergent numerical results for the first five natural frequencies of the pulley-belt dynamical system. In the following numerical examples, the first 16 modes are used for Galerkin truncation method if there is no clarification.

4.1.2. Parametric Studies. Figure 6 describes the effects of the physical parameters and the initial static tension on the first five natural frequencies of the two-pulley belt-driven system. Figures 6(a) and 6(b) show that the effects of the initial static tension on the fourth and fifth modes are greater than on the first three modes. As it is shown in Figures 6(c), 6(d), 6(e), and 6(f), the first five natural frequencies of the pulleybelt system are very sensitive to Young's modulus and the height of the belt. Particularly, the fourth and fifth natural frequencies are more sensitive to Young's modulus. Since EI accounts for the bending stiffness of the belt, the numerical results illustrate that the belt bending stiffness significantly influences the first five natural frequencies of the pulley-belt system. Figures $6(\mathrm{~g})$ and $6(\mathrm{~h})$ depict the effects of the stiffness of the wrap spring between driven pulley and the accessory 


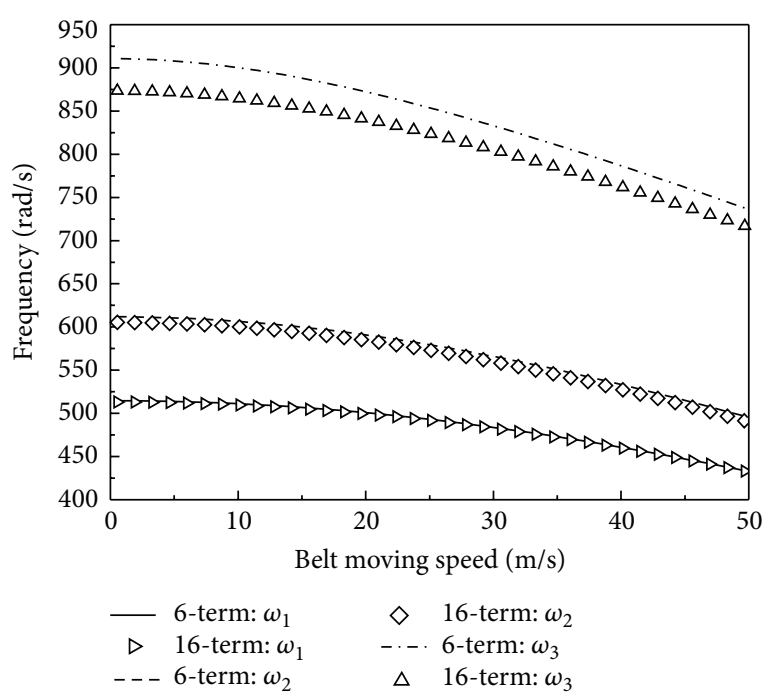

(a) The first three modes: 6-term versus 16-term

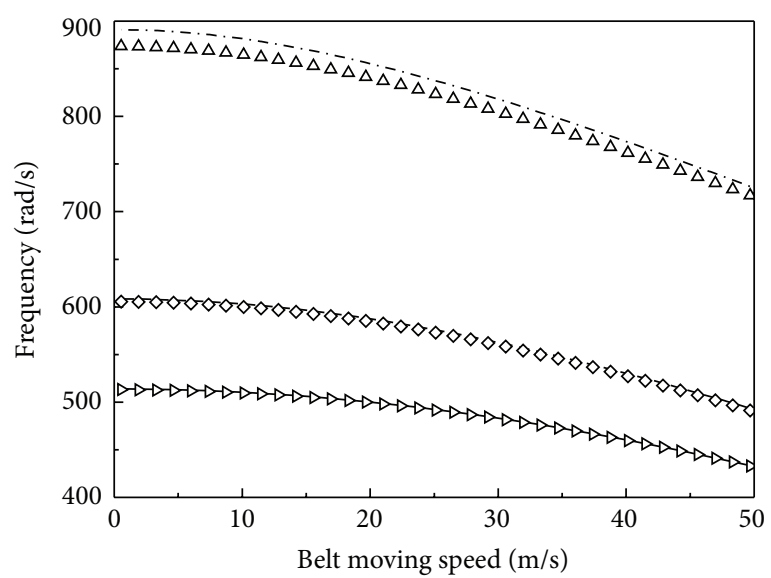

$\triangleright$ 16-term: $\omega_{1} \quad \ldots$ 8-term: $\omega_{3}$

- - 8-term: $\omega_{2} \quad \Delta \quad$ 16-term: $\omega_{3}$

(c) The first three modes: 8 -term versus 16-term

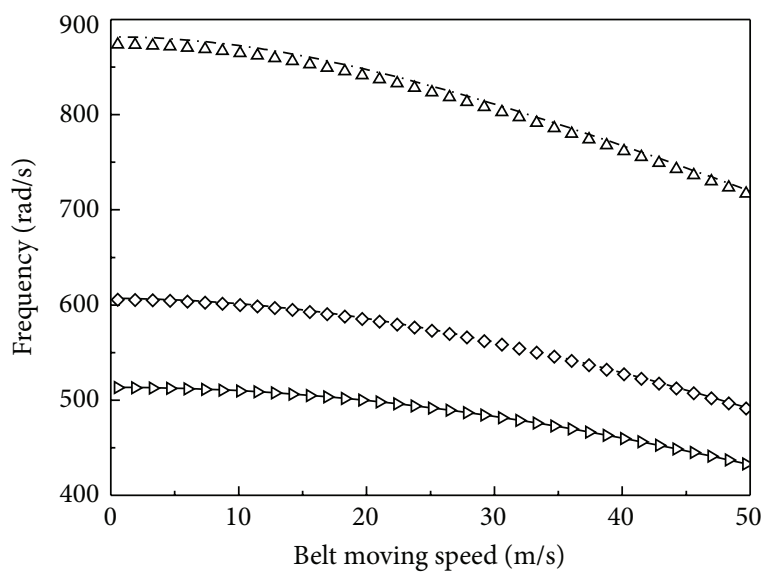

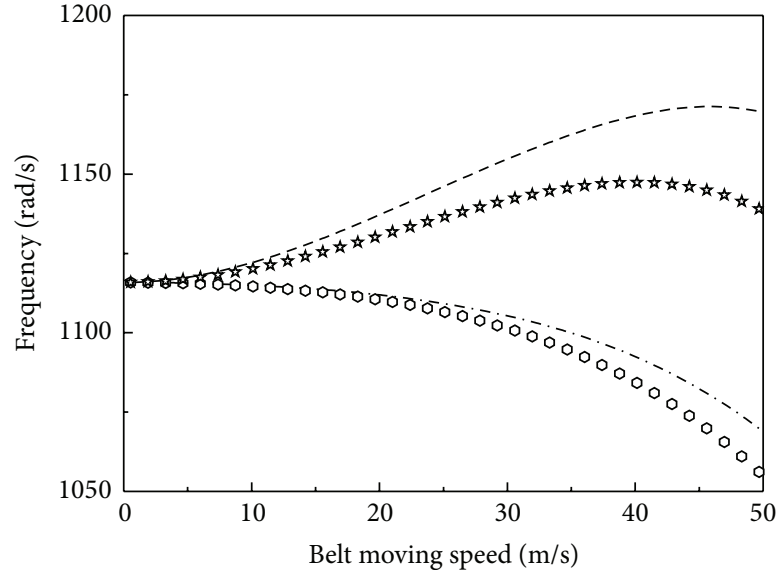

-.-. 6-term: $\omega_{4} \quad$--- 6-term: $\omega_{5}$

- 16-term: $\omega_{4}$ \ 16-term: $\omega_{5}$

(b) The fourth and fifth modes: 6-term versus 16-term

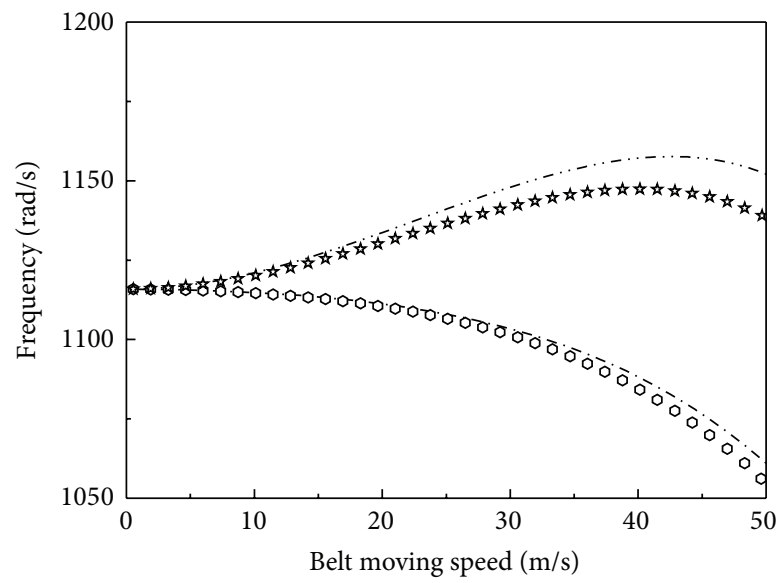

-.- 8-term: $\omega_{4} \quad \cdots-\cdots \quad$ 8-term: $\omega_{5}$

(d) The fourth and fifth modes: 8 -term versus 16-term

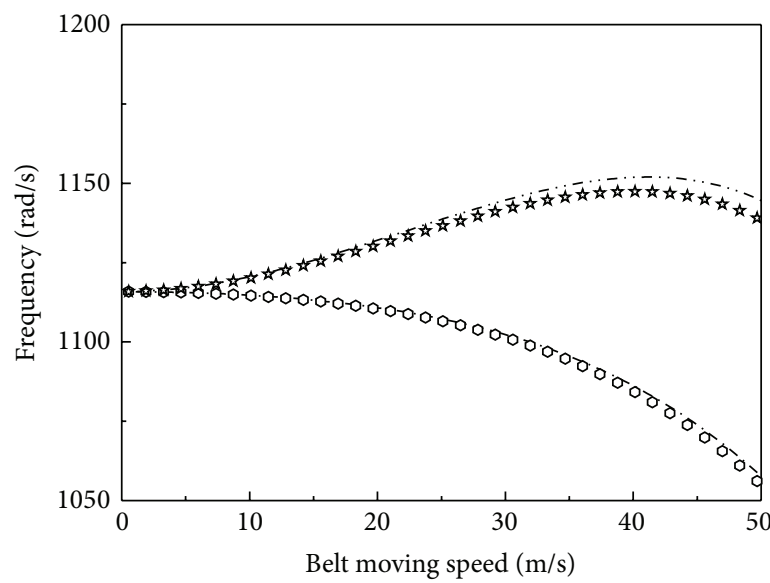

-. - 10-term: $\omega_{4} \quad-\cdots-10$-term: $\omega_{5}$

- 16-term: $\omega_{4}$ \ 16-term: $\omega_{5}$

(f) The fourth and fifth modes: 10 -term versus 16 -term

(e) The first three modes: 10 -term versus 16 -term

FIgure 5: Continued. 


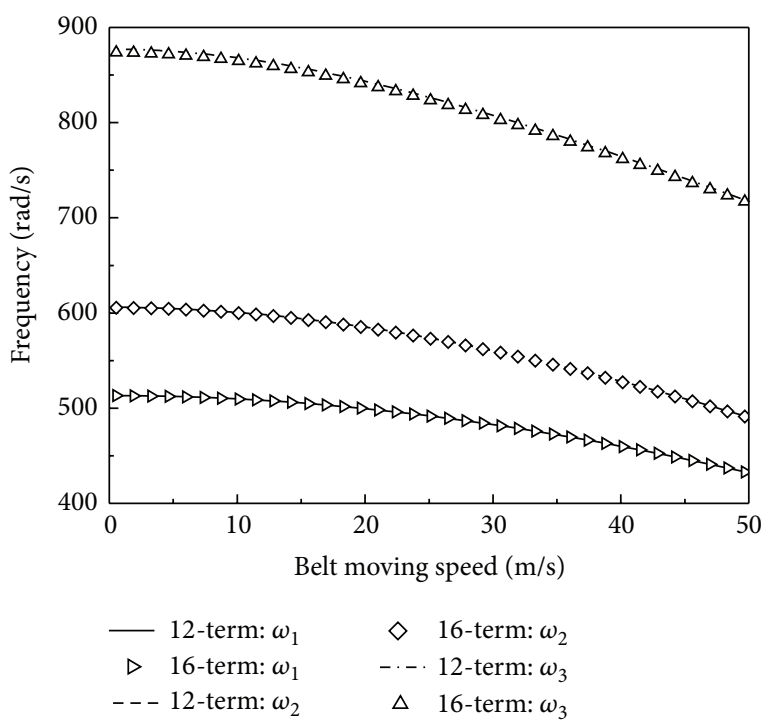

(g) The first three modes: 12 -term versus 16 -term

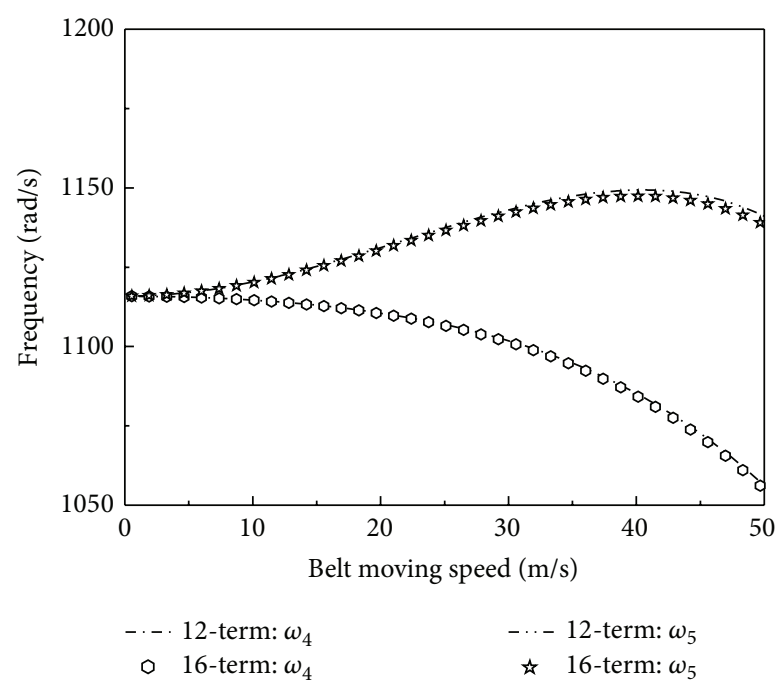

(h) The fourth and fifth modes: 12-term versus 16-term

FIGURE 5: The comparisons of the natural frequencies for different truncation terms.

shaft on the first five natural frequencies of the two-pulley belt-driven system. As shown in Figures 6(g) and 6(h), the wrap spring stiffness only influences the third and the fifth natural frequencies. The comparisons in Figure 6 show that the first five natural frequencies of the dynamical system increase with increasing initial static tension, Young's modulus, and height of the belt. Meanwhile, only the third and the fifth modes increase with increasing stiffness of the wrap spring.

4.2. Quadrature Methods. In this section, the differential and integral quadrature methods are applied to determine the first several natural frequencies for the discrete-continuous system. Substituting (12) into (8) and (9) yields a series of ordinary differential equations

$$
\begin{aligned}
& \ddot{u}_{i j}+ 2 c \sum_{k=1}^{N} A_{j k}^{(1)} \dot{u}_{i k} \\
&+\sum_{k=1}^{N}\left[\left(c^{2}-1-\widehat{T}\right) A_{j k}^{(2)}+k_{f}^{2} A_{j k}^{(4)}\right] u_{i k} \\
&-k_{1} N_{i j} \sum_{k=1}^{N}\left[u_{i k} \sum_{g=1}^{N} I_{g} \widehat{w}_{i g}^{\prime} A_{g k}^{(1)}\right] \\
&+N_{i j} \tilde{\lambda}_{i}=0, \quad j=3,4, \ldots, N-2, \\
& \sum_{k=1}^{N} A_{2 k}^{(2)} u_{i k}=\sum_{k=1}^{N} A_{N-1 k}^{(2)} u_{i k}=0,
\end{aligned}
$$

$$
\begin{gathered}
J \ddot{\vartheta}_{1}-k_{1} \sum_{k=1}^{N}\left[u_{1 k} \sum_{g=1}^{N} I_{g} \widehat{w}_{1 g}^{\prime} A_{g k}^{(1)}\right] \\
+k_{1} \sum_{k=1}^{N}\left[u_{2 k} \sum_{g=1}^{N} I_{g} \widehat{w}_{2 g}^{\prime} A_{g k}^{(1)}\right] \\
+\left(\frac{2 k_{1} r}{l}+\frac{r_{a} k_{d}}{r}\right) \vartheta_{1}-\frac{2 k_{1} r}{l} \vartheta_{2}-\frac{r_{a} k_{d}}{r} \vartheta_{a}=0 \\
J \ddot{\vartheta}_{2}+k_{1} \sum_{k=1}^{N}\left[u_{1 k} \sum_{g=1}^{N} I_{g} \widehat{w}_{1 g}^{\prime} A_{g k}^{(1)}\right] \\
-k_{1} \sum_{k=1}^{N}\left[u_{2 k} \sum_{g=1}^{N} I_{g} \widehat{w}_{2 g}^{\prime} A_{g k}^{(1)}\right] \\
-\frac{2 k_{1} r}{l} \vartheta_{1}+\frac{2 k_{1} r}{l} \vartheta_{2}=0 \\
J_{a} \ddot{\vartheta}_{a}-k_{d} \vartheta_{1}+k_{d} \vartheta_{a}=0
\end{gathered}
$$

where

$$
N_{i j}=\sum_{k=1}^{N} A_{j k}^{(2)} \widehat{w}_{i k}, \quad i=1,2 .
$$

Suppose that the solution to (35) takes the form

$$
\begin{aligned}
u_{i j}(t)=q_{i j} e^{i \omega t}, \quad i=1,2 ; j=1,2, \ldots, N, & \\
\vartheta_{1} & =Q_{1} e^{i \omega t}, \\
\vartheta_{2} & =Q_{2} e^{i \omega t}, \\
\vartheta_{a} & =Q_{a} e^{i \omega t} .
\end{aligned}
$$




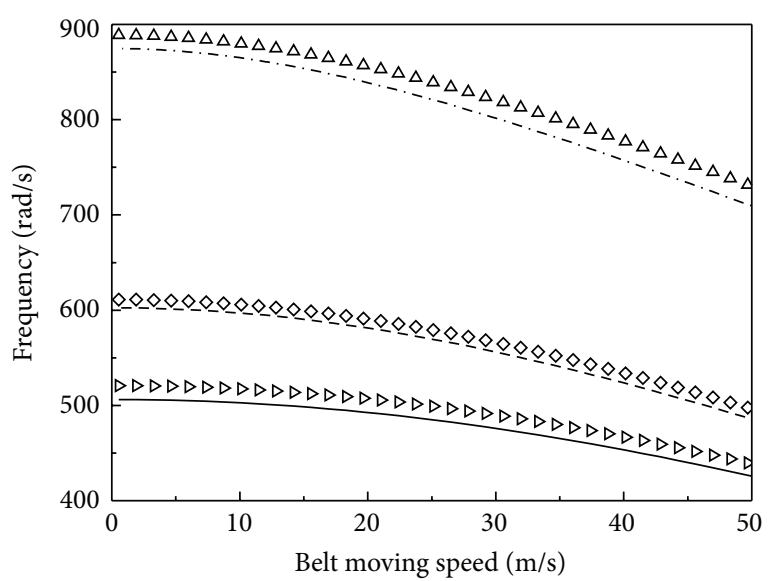
- $P_{0}=300: \omega_{1}$
$\triangleright P_{0}=400: \omega_{1}$
- - $P_{0}=300: \omega_{2}$
$\diamond P_{0}=400: \omega_{2}$
$\cdots P_{0}=300: \omega_{3}$
$\triangle P_{0}=400: \omega_{3}$

(a) The first three modes: initial static tension

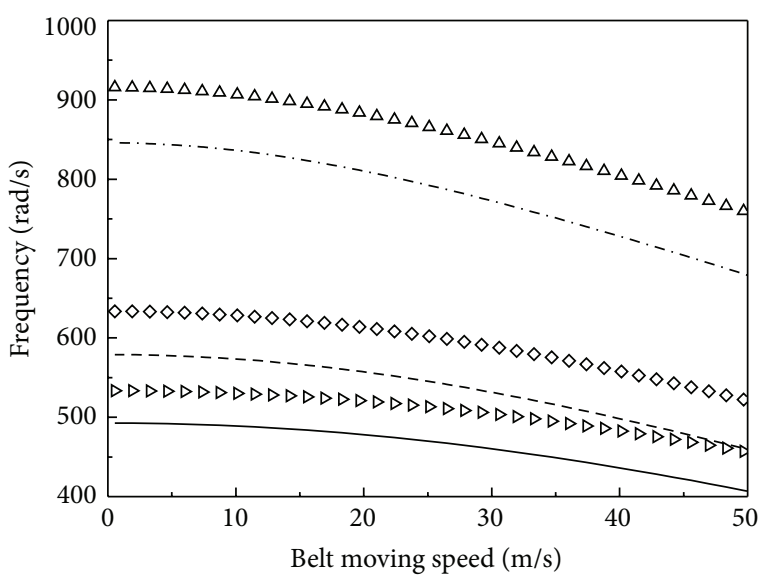

$E=1.8 * 10^{9}: \omega_{1}$

$\triangleright E=2.2 * 10^{9}: \omega_{1}$ $---E=1.8 * 10^{9}: \omega_{2}$

$\diamond \quad E=2.2 * 10^{9}: \omega_{2}$

$\rightarrow \cdot \cdot E=1.8 * 10^{9}: \omega_{3}$

$\triangle \quad E=2.2 * 10^{9}: \omega_{3}$

(c) The first three modes: Young's modulus

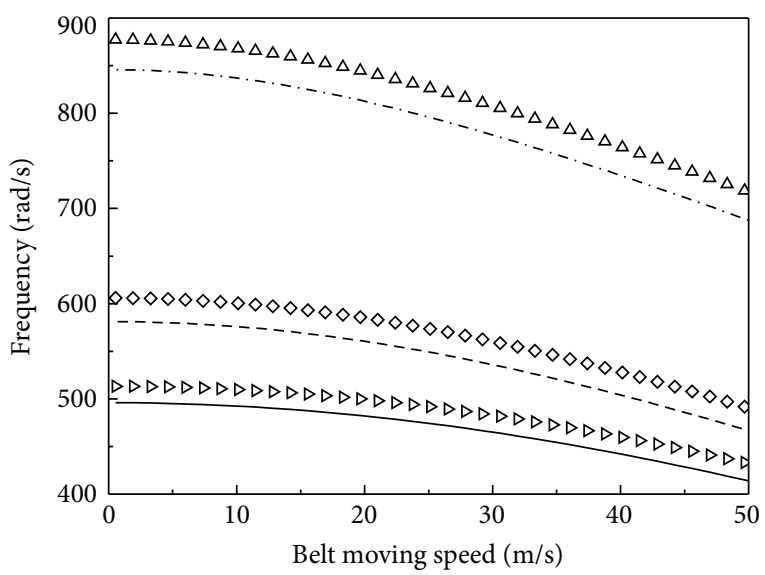

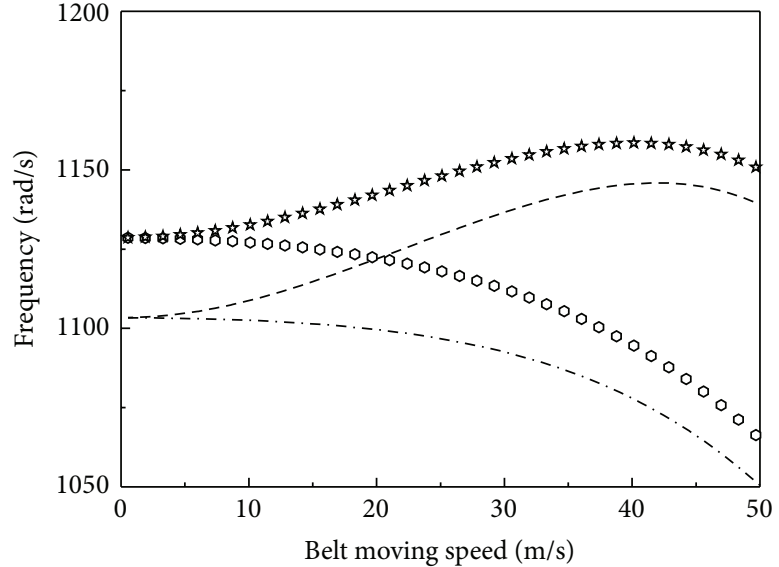

$-\cdot \cdot P_{0}=300: \omega_{4}$

- $P_{0}=400: \omega_{4}$

$---P_{0}=300: \omega_{5}$

\& $P_{0}=400: \omega_{5}$

(b) The fourth and fifth modes: initial static tension

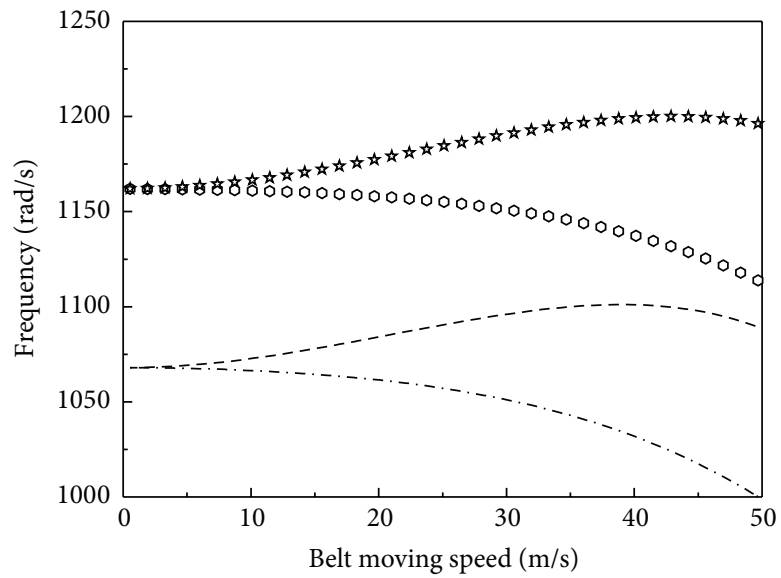

-.. $E=1.8 * 10^{9}: \omega_{4}$

- $E=2.2 * 10^{9}: \omega_{4}$

-- $E=1.8 * 10^{9}: \omega_{5}$ मt $E=2.2 * 10^{9}: \omega_{5}$

(d) The fourth and fifth modes: Young's modulus

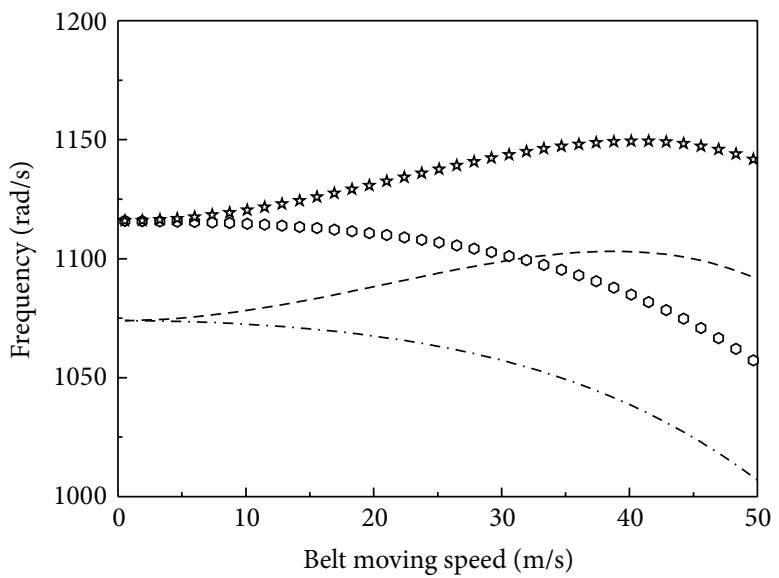

-.. $h=0.0048: \omega_{4}$

○ $h=0.005: \omega_{4}$

$---h=0.0048: \omega_{5}$

$h=0.005: \omega_{5}$

(f) The fourth and fifth modes: the belt's height

(e) The first three modes: the belt's height

Figure 6: Continued. 

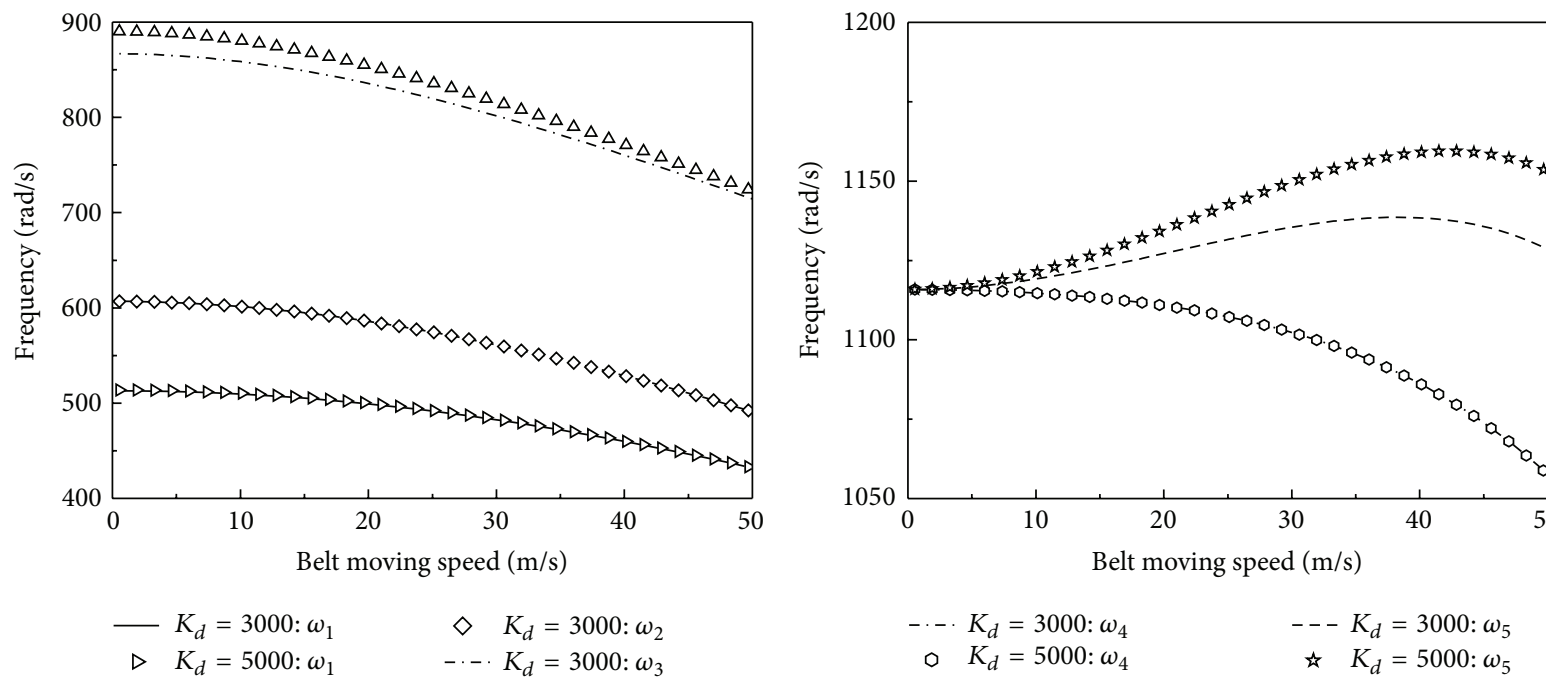

$$
\begin{array}{rlrl}
\longrightarrow K_{d} & =3000: \omega_{1} & \diamond & K_{d}=3000: \omega_{2} \\
\triangleright \quad K_{d} & =5000: \omega_{1} & -\cdot-\cdot K_{d}=3000: \omega_{3} \\
---K_{d} & =3000: \omega_{2} & \Delta & K_{d}=5000: \omega_{3}
\end{array}
$$

(g) The first three modes: stiffness of wrap spring (h) The fourth and fifth modes: stiffness of wrap spring

FIgURE 6: The parametric studies for the natural frequencies.

Substituting (37) into (35) yields

$$
\begin{aligned}
& -\omega^{2} q_{i j}+2 i c \omega \sum_{k=1}^{N} A_{j k}^{(1)} q_{i k} \\
& +\sum_{k=1}^{N}\left[\left(c^{2}-1-\widehat{T}\right) A_{j k}^{(2)}\right. \\
& \left.+k_{f}^{2} A_{j k}^{(4)}-k_{1} \widehat{w}_{i j}^{\prime \prime} \sum_{g=1}^{N} I_{g} \widehat{w}_{i g}^{\prime} A_{g k}^{(1)}\right] \\
& \times q_{i k}+\widehat{w}_{i j}^{\prime \prime} \tilde{\lambda}_{i}=0, \quad j=3,4, \ldots, N-2, \\
& q_{i 1}=q_{i N}=0 \\
& \sum_{k=1}^{N} A_{2 k}^{(2)} q_{i k}=\sum_{k=1}^{N} A_{N-1 k}^{(2)} q_{i k}=0 \\
& -\omega^{2} J Q_{1}=k_{1} \sum_{k=1}^{N}\left[q_{1 k} \sum_{g=1}^{N} I_{g} \widehat{w}_{1 g}^{\prime} A_{g k}^{(1)}\right] \\
& -k_{1} \sum_{k=1}^{N}\left[q_{2 k} \sum_{g=1}^{N} I_{g} \widehat{w}_{2 g}^{\prime} A_{g k}^{(1)}\right] \\
& -\left(\frac{2 k_{1} r}{l}+\frac{r_{a} k_{d}}{r}\right) \vartheta_{1}+\frac{2 k_{1} r}{l} \vartheta_{2}+\frac{r_{a} k_{d}}{r} \vartheta_{a} \text {, } \\
& -\omega^{2} J Q_{2}=-k_{1} \sum_{k=1}^{N}\left[q_{1 k} \sum_{g=1}^{N} I_{g} \widehat{w}_{1 g}^{\prime} A_{g k}^{(1)}\right] \\
& +k_{1} \sum_{k=1}^{N}\left[q_{2 k} \sum_{g=1}^{N} I_{g} \widehat{w}_{2 g}^{\prime} A_{g k}^{(1)}\right] \\
& +\frac{2 k_{1} r}{l} \vartheta_{1}-\frac{2 k_{1} r}{l} \vartheta_{2} \\
& -\omega^{2} J_{a} Q_{a}=k_{d} \vartheta_{1}-k_{d} \vartheta_{a} \text {. }
\end{aligned}
$$

Equation (38) also can be written in matrix-vector form as (32) with all coefficients $M_{h k}, G_{h k}$, and $K_{h k}, h, k=$ $1,2, \ldots, 2 N+3$ are determined by quadrature methods [36, 37]. Similarly, the natural frequencies are determined in a similar way to the previous case with Galerkin truncation. Therefore, the natural frequencies of the present dynamical system can be obtained from (34) based on determinant of coefficients of (32) to be zero.

The first five natural frequencies of the pulley-belt system coupled with accessory are shown in Figure 7 based on the differential and integral quadrature methods. Furthermore, the natural frequencies from these quadrature methods are compared for three different numbers of sampling points. The numerical results illustrate that the quadrature methods with sampling points, $N=17$ and 19, deliver the same result. Meanwhile, there is discernible difference between the fourth and fifth natural frequencies based on $N=15$ and 17. Therefore, the numerical results in Figure 7 exhibit that the quadrature methods have good convergence properties for predicting the free vibration characteristics for the present pulley-belt system. Moreover, the quadrature methods with $N=17$ are adopted in the following numerical examples.

In order to verify the validity of the Galerkin method in Section 4.1, Figure 8 describes the comparisons of the first five natural frequencies of the present dynamical system via the quadrature methods and the 12-term and 16-term Galerkin truncation. As it is seen from Figure 8(a), the first two natural frequencies, which are predicted by using the 12-term Galerkin truncation, are very close to that of the quadrature methods. Meanwhile, Figure $8(\mathrm{~b})$ shows that the third, fourth, and fifth natural frequencies predicted via the 12-term truncation are slightly larger that of the quadrature methods. Nevertheless, Figures 8(c) and 8(d) illustrate that the first five natural frequencies via the 16-term Galerkin truncation are very close to those via the quadrature methods. 


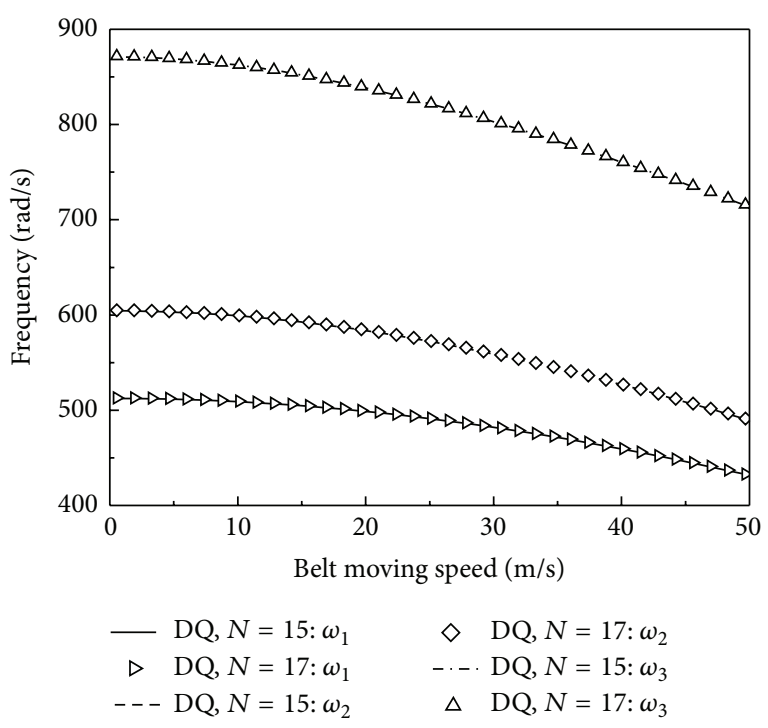

(a) The first three modes: $N=15$ versus $N=17$

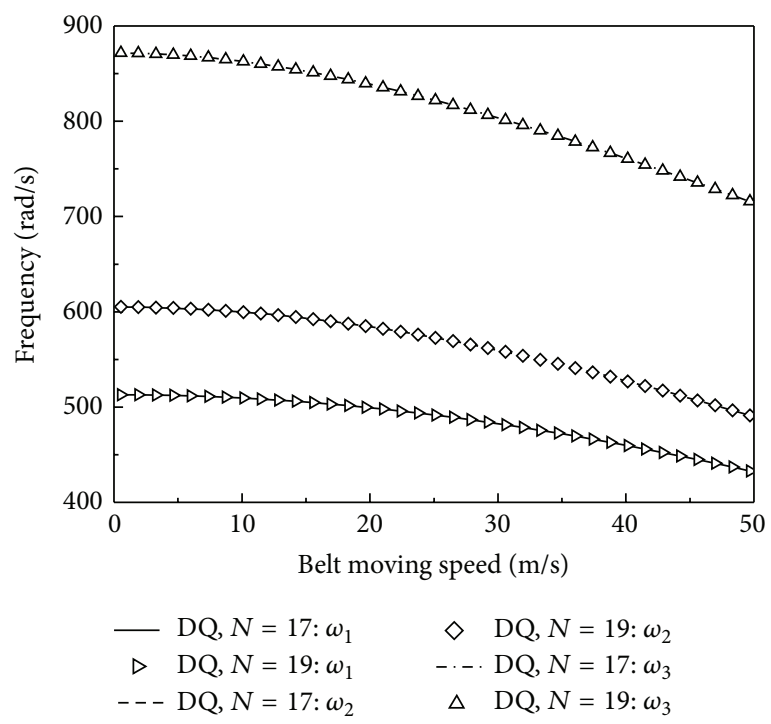

(c) The first three modes: $N=17$ versus $N=19$

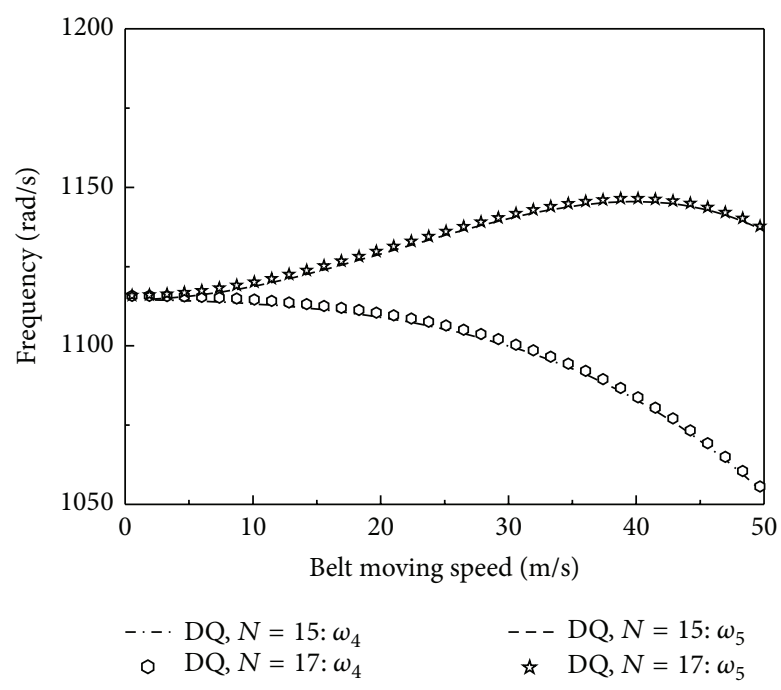

(b) The fourth and fifth modes: $N=15$ versus $N=17$

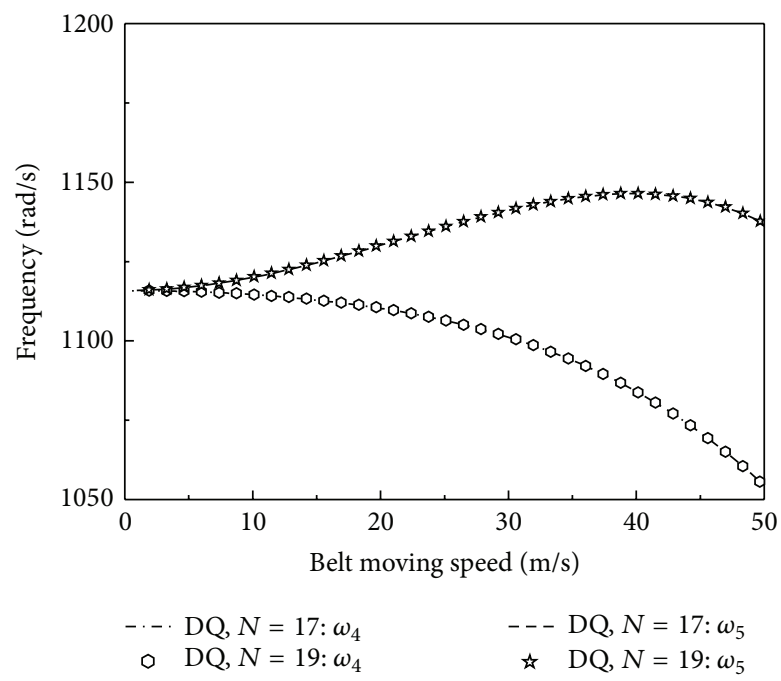

(d) The fourth and fifth modes: $N=17$ versus $N=19$

FIGURE 7: Convergence of the nontrivial equilibrium solutions: the number of sampling points.

Therefore, the numerical results of the first five natural frequencies via the 16-term truncation are confirmed by the quadrature methods.

\section{Conclusions}

The equilibria and the free vibration characteristics of a two-pulley belt-driven system connected with greater weight accessory are studied in the present work. Considering the effects of the bending stiffness of the translating belt, the belt is modeled as an axially moving viscoelastic beam. A nonlinear piecewise discrete-continuous model is established for coupling the transverse vibration of the translating belt and the rotation vibration of the pulleys and accessory. The nontrivial equilibriums of the belt are, respectively, numerically calculated by the viscoelastic model and an iterative scheme based on the equilibrium equation. Furthermore, by introducing a coordinate transform, new equations are derived for governing the vibration near the nontrivial equilibrium. The natural frequencies of the pulley-belt system are, respectively, studied via the high-order Galerkin method as well as the differential and integral quadrature methods. The following major conclusions are drawn from this study.

(1) The nontrivial equilibrium solutions of the translating belt spans and the first five natural frequencies of the dynamical system are both very sensitive to the belt bending stiffness.

(2) The first five natural frequencies of the pulley-belt system are increasing with the initial static tension, Young's modulus, and the height of the belt. On the 


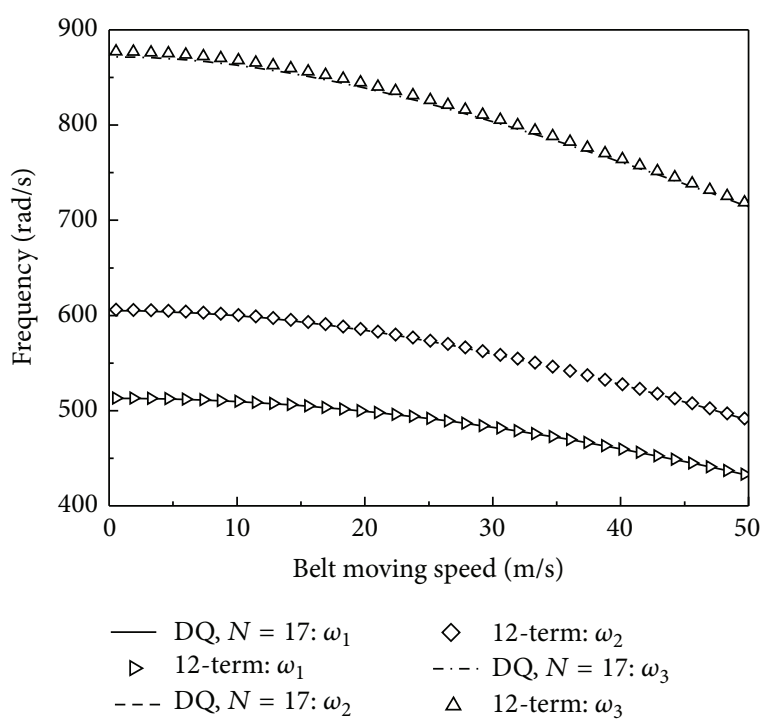

(a) The first three modes: 12-term versus DQM and IQM

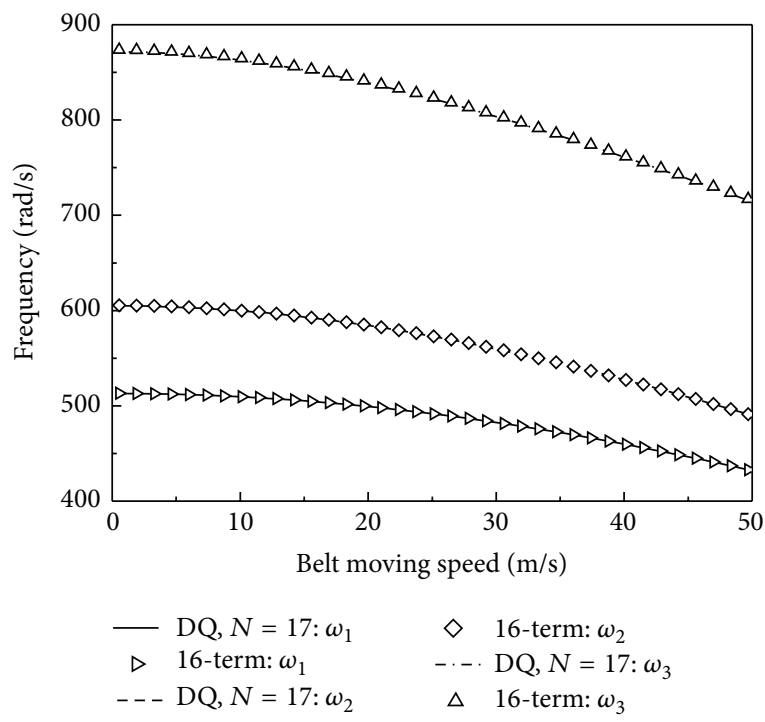

(c) The first three modes: 16-term versus DQM and IQM

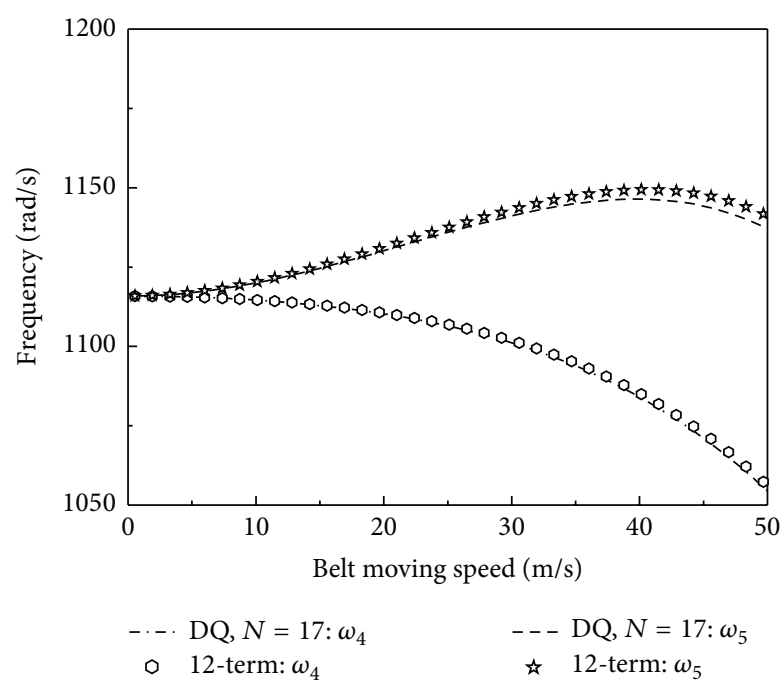

(b) The fourth and fifth modes: 12 -term versus quadrature methods

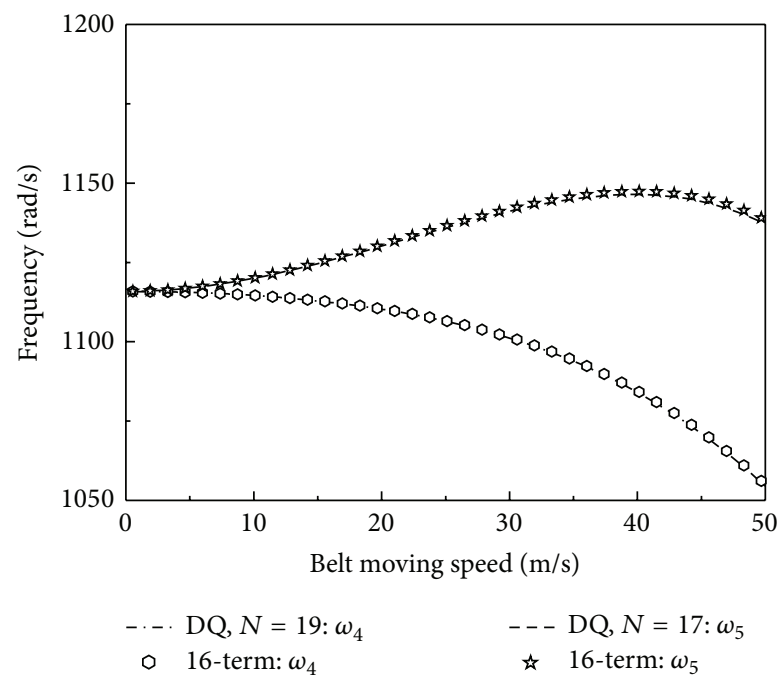

(d) The fourth and fifth modes: 16-term versus quadrature methods

FIgURE 8: The comparisons of the natural frequencies via the Galerkin truncation and the quadrature methods.

other hand, only the third and the fifth modes are influenced by the stiffness of the wrap spring.

(3) The numerical results demonstrate that 16-term Galerkin truncation delivers the convergent results for the first five natural frequencies of the pulley-belt system. Moreover, the first five natural frequencies, which are predicted by using the 16-term Galerkin truncation and the quadrature methods, are almost the same.

\section{Conflict of Interests}

The authors declare that there is no conflict of interests regarding the publication of this paper.

\section{Acknowledgments}

The authors gratefully acknowledge the support of the State Key Program of National Natural Science Foundation of China through Grant no. 11232009 and the National Natural Science Foundation of China through Grant nos. 11372171, 11272166, and 11002075.

\section{References}

[1] A. G. Ulsoy, J. E. Whitesell, and M. D. Hooven, "Design of belttensioner systems for dynamic stability," Journal of Vibration, Acoustics, Stress, and Reliability in Design, vol. 107, no. 3, pp. 282290, 1985.

[2] S. Abrate, "Vibrations of belts and belt drives," Mechanism and Machine Theory, vol. 27, no. 6, pp. 645-659, 1992. 
[3] L.-Q. Chen, "Analysis and control of transverse vibrations of axially moving strings," Applied Mechanics Reviews, vol. 58, no. 1-6, pp. 91-116, 2005.

[4] C. D. Mote Jr. and W. Z. Wu, "Vibration coupling in continuous belt and band systems," Journal of Sound and Vibration, vol. 102, no. 1, pp. 1-9, 1985.

[5] L. Kong and R. G. Parker, "Equilibrium and belt-pulley vibration coupling in serpentine belt drives," Transactions ASME, Journal of Applied Mechanics, vol. 70, no. 5, pp. 739-750, 2003.

[6] L. Kong and R. G. Parker, "Coupled belt-pulley vibration in serpentine drives with belt bending stiffness," Journal of Applied Mechanics, vol. 71, no. 1, pp. 109-119, 2004.

[7] L. Zhang and J. W. Zu, "One-to-one auto-parametric resonance in serpentine belt drive systems," Journal of Sound and Vibration, vol. 232, no. 4, pp. 783-806, 2000.

[8] P. Vernay, G. Ferraris, A. Delbez, and P. Ouplomb, “Transient behaviour of a sprag-type over-running clutch: an experimental study," Journal of Sound and Vibration, vol. 248, no. 3, pp. 567$572,2001$.

[9] M. J. Leamy and T. M. Wasfy, "Transient and steady-state dynamic finite element modeling of belt-drives," Journal of Dynamic Systems, Measurement and Control, vol. 124, no. 4, pp. 575-581, 2002.

[10] F. Zhu and R. G. Parker, "Non-linear dynamics of a one-way clutch in belt-pulley systems," Journal of Sound and Vibration, vol. 279, no. 1-2, pp. 285-308, 2005.

[11] F. Zhu and R. G. Parker, "Perturbation analysis of a clearancetype nonlinear system," Journal of Sound and Vibration, vol. 292, no. 3-5, pp. 969-979, 2006.

[12] E. M. Mockensturm and R. Balaji, "Piece-wise linear dynamic systems with one-way clutches," Transactions of the ASME, Journal of Vibration and Acoustics, vol. 127, no. 5, pp. 475-482, 2005.

[13] R. Balaji and E. M. Mockensturm, "Dynamic analysis of a frontend accessory drive with a decoupler/isolator," International Journal of Vehicle Design, vol. 39, no. 3, pp. 208-231, 2005.

[14] C. Gill-Jeong, "Nonlinear behavior analysis of spur gear pairs with a one-way clutch," Journal of Sound and Vibration, vol. 301, no. 3-5, pp. 760-776, 2007.

[15] K. W. Wang and C. D. Mote Jr., "Vibration coupling analysis of band/wheel mechanical systems," Journal of Sound and Vibration, vol. 109, no. 2, pp. 237-258, 1986.

[16] R. S. Beikmann, N. C. Perkins, and A. G. Ulsoy, "Free vibration of serpentine belt drive systems," Journal of Vibration and Acoustics, vol. 118, no. 3, pp. 406-413, 1996.

[17] F. Zhu and R. G. Parker, "Piece-wise linear dynamic analysis of serpentine belt drives with a one-way clutch," Proceedings of the Institution of Mechanical Engineers, Part C: Journal of Mechanical Engineering Science, vol. 222, no. 7, pp. 1165-1176, 2008.

[18] H. Ding and J. W. Zu, "Effect of one-way clutch on the nonlinear vibration of belt-drive systems with a continuous belt model," Journal of Sound and Vibration, vol. 332, no. 24, pp. 6472-6487, 2013.

[19] R. K. Jha and R. G. Parker, "Spatial discretization of axially moving media vibration problems," Journal of Vibration and Acoustics, Transactions of the ASME, vol.122, no. 3, pp. 290-294, 2000 .
[20] F. Pellicano and F. Zirilli, "Boundary layers and non-linear vibrations in an axially moving beam," International Journal of Non-Linear Mechanics, vol. 33, no. 4, pp. 691-711, 1998.

[21] F. Pellicano and F. Vestroni, "Complex dynamics of high-speed axially moving systems," Journal of Sound and Vibration, vol. 258, no. 1, pp. 31-44, 2002.

[22] L. Kong and R. G. Parker, "Mechanics of serpentine belt drives with tensioner assemblies and belt bending stiffness," Journal of Mechanical Design, Transactions of the ASME, vol. 127, no. 5, pp. 957-966, 2005.

[23] K. Dufva, K. Kerkkänen, L. G. Maqueda, and A. A. Shabana, "Nonlinear dynamics of three-dimensional belt drives using the finite-element method," Nonlinear Dynamics, vol. 48, no. 4, pp. 449-466, 2007.

[24] F. Zhu and R. G. Parker, "Influence of tensioner dry friction on the vibration of belt drives with belt bending stiffness," Journal of Vibration and Acoustics, vol. 130, no. 1, Article ID 011002, 2008.

[25] P. R. Scurtu, M. Clark, and J. W. Zu, "Coupled longitudinal and transverse vibration of automotive belts under longitudinal excitations using analog equation method," Journal of Vibration and Control, vol. 18, no. 9, pp. 1336-1352, 2012.

[26] H. Ding and L.-Q. Chen, "Galerkin methods for natural frequencies of high-speed axially moving beams," Journal of Sound and Vibration, vol. 329, no. 17, pp. 3484-3494, 2010.

[27] H. Ding, G.-C. Zhang, L.-Q. Chen, and S.-P. Yang, "Forced vibrations of supercritically transporting viscoelastic beams," Transactions of the ASME, Journal of Vibration and Acoustics, vol. 134, no. 5, Article ID 051007, pp. 1-11, 2012.

[28] P. Malekzadeh and A. R. Vosoughi, "DQM large amplitude vibration of composite beams on nonlinear elastic foundations with restrained edges," Communications in Nonlinear Science and Numerical Simulation, vol. 14, no. 3, pp. 906-915, 2009.

[29] M. Mallakzadeh, A. A. Pasha Zanoosi, and A. Alibeigloo, "Fundamental frequency analysis of microtubules under different boundary conditions using differential quadrature method," Communications in Nonlinear Science and Numerical Simulation, vol. 18, no. 8, pp. 2240-2251, 2013.

[30] M. J. Leamy, "On a perturbation method for the analysis of unsteady belt-drive operation," Journal of Applied Mechanics, Transactions ASME, vol. 72, no. 4, pp. 570-580, 2005.

[31] G. Čepon and M. Boltežar, "Dynamics of a belt-drive system using a linear complementarity problem for the belt-pulley contact description," Journal of Sound and Vibration, vol. 319, no. 3-5, pp. 1019-1035, 2009.

[32] H. Ding, G. C. Zhang, and L. Q. Chen, "Supercritical equilibrium solutions of axially moving beams with hybrid boundary conditions," Mechanics Research Communications, vol. 38, no. 1, pp. 52-56, 2011.

[33] H. Ding, G. C. Zhang, and L. Q. Chen, "Supercritical vibration of nonlinear coupled moving beams based on discrete Fourier transform," International Journal of Non-Linear Mechanics, vol. 47, no. 10, pp. 1095-1104, 2012.

[34] B. Kang and C. A. Tan, "Nonlinear response of a beam under distributed moving contact load," Communications in Nonlinear Science and Numerical Simulation, vol. 11, no. 2, pp. 203-232, 2006.

[35] X.-J. Li and L.-Q. Chen, "Modal analysis of coupled vibration of belt drive systems," Applied Mathematics and Mechanics (English Edition), vol. 29, no. 1, pp. 9-13, 2008. 
[36] H. Ding and J. W. Zu, "Periodic and chaotic responses of an axially accelerating viscoelastic beam under two-frequency excitations," International Journal of Applied Mechanics, vol. 5, no. 2, Article ID 1350019, 2013.

[37] K. Daneshjou, M. Talebitooti, and R. Talebitooti, "Free vibration and critical speed of moderately thick rotating laminated composite conical shell using generalized differential quadrature method," Applied Mathematics and Mechanics (English Edition), vol. 34, no. 4, pp. 437-456, 2013. 


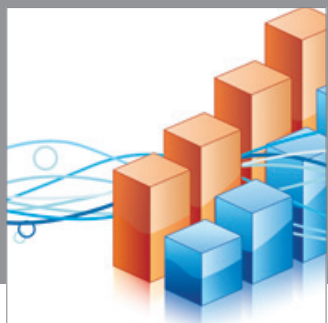

Advances in

Operations Research

mansans

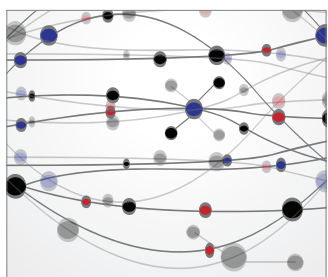

The Scientific World Journal
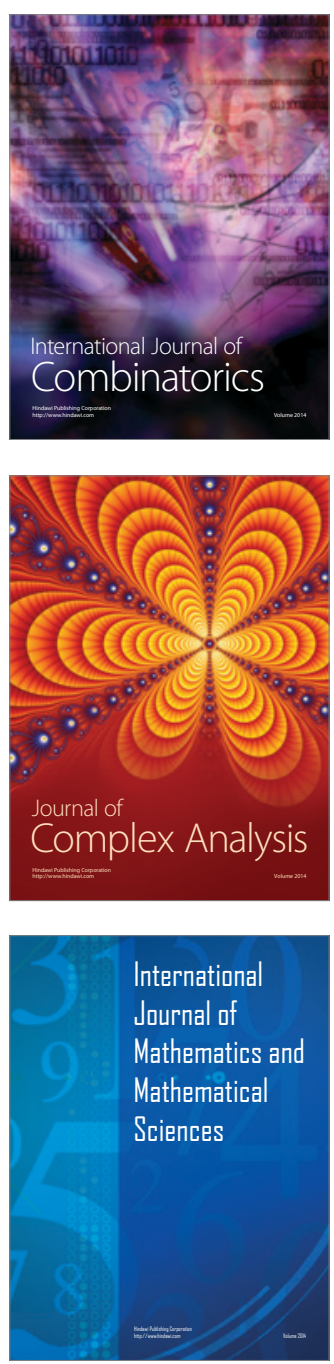
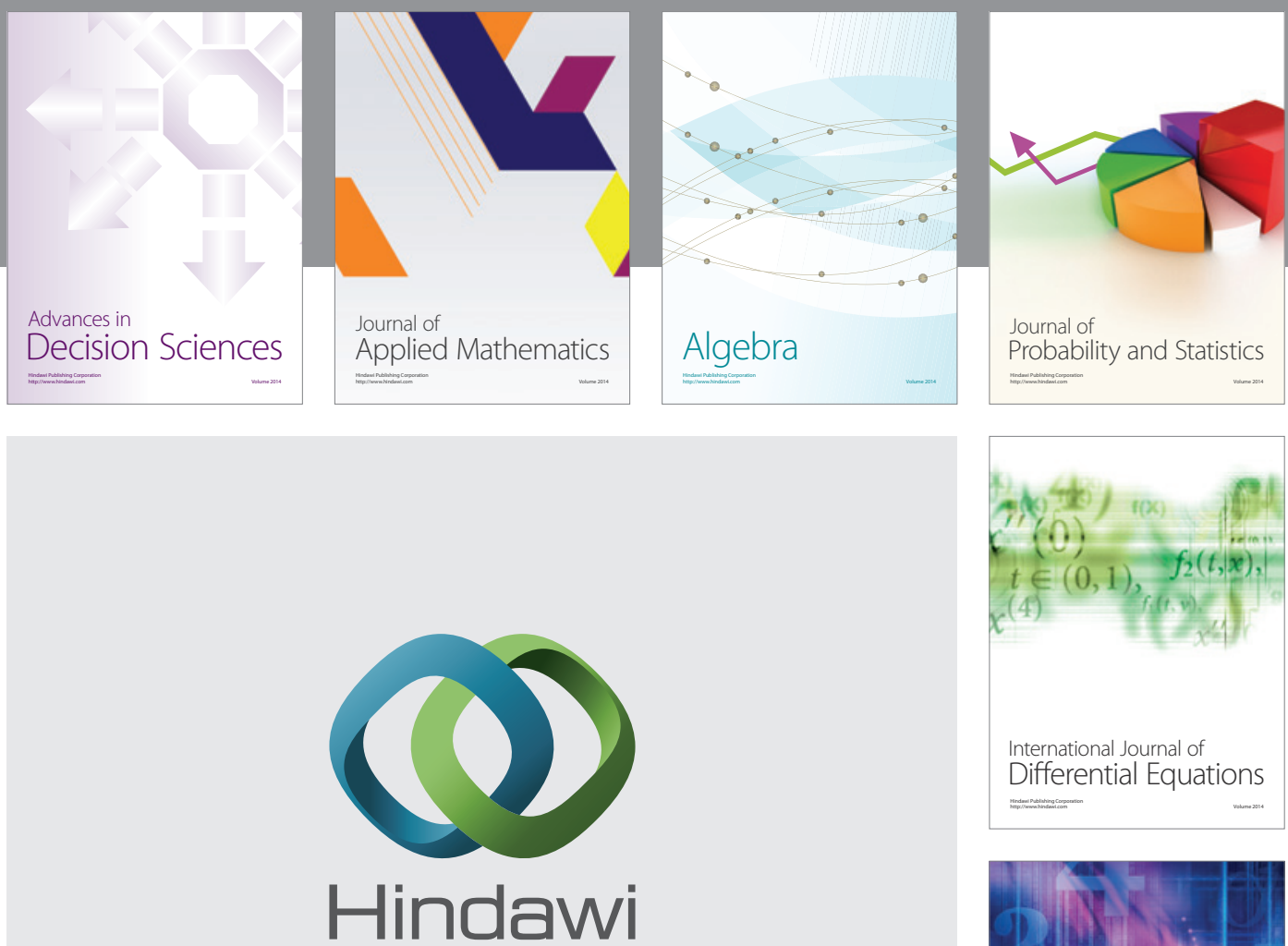

Submit your manuscripts at http://www.hindawi.com
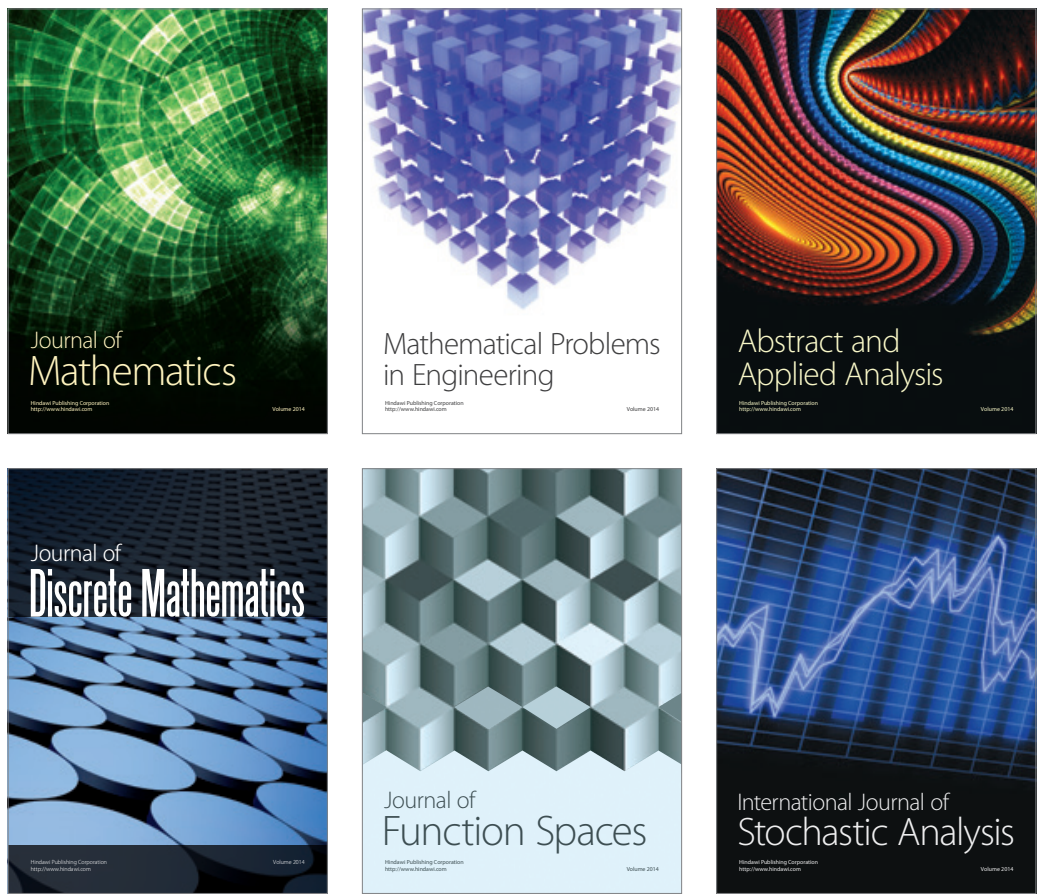

Journal of

Function Spaces

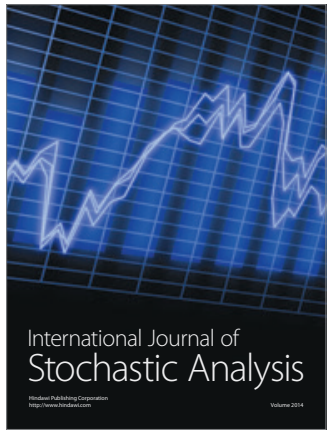

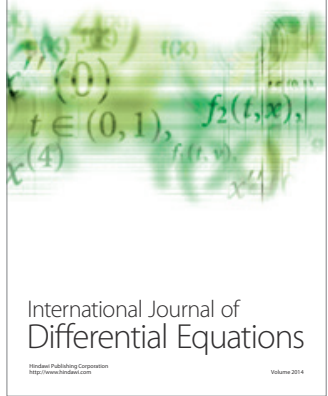
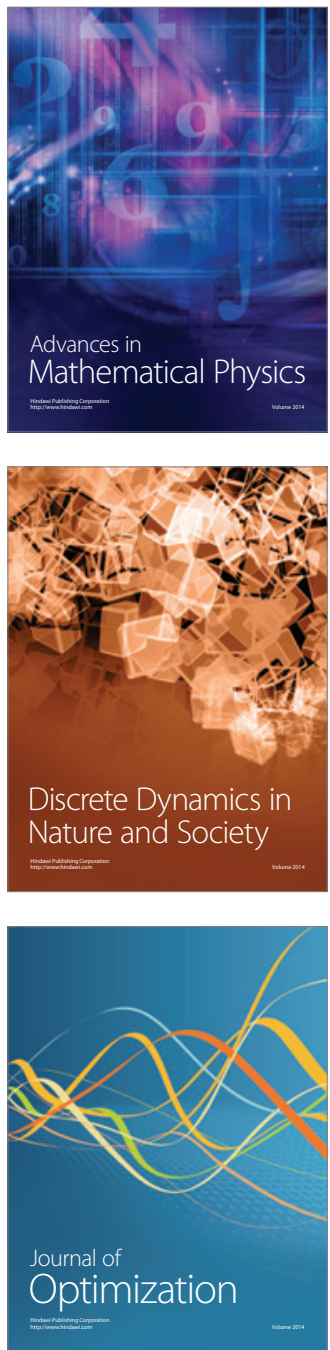